Patricia Arena Abramides

\title{
Avaliação sequencial do equilíbrio pré e pós-implante coclear em pacientes com surdez pós-lingual
}

\author{
Tese apresentada à Faculdade de Medicina \\ da Universidade de São Paulo para \\ obtenção do título de Doutor em Ciências \\ Programa de Otorrinolaringologia \\ Orientadora: Prof ${ }^{a}$. Dra. Roseli Saraiva \\ Moreira Bittar
}

Versão Corrigida

São Paulo

2014 
Dados Internacionais de Catalogação na Publicação (CIP)

Preparada pela Biblioteca da

Faculdade de Medicina da Universidade de São Paulo

Creprodução autorizada pelo autor

\begin{abstract}
Abramides, Patricia Arena
Avaliação sequencial do equilíbrio pré e pós implante coclear em pacientes com surdez pós-lingual / Patricia Arena Abramides. -- São Paulo, 2014.

Tese(doutorado)--Faculdade de Medicina da Universidade de São Paulo. Programa de Otorrinolaringologia.

Orientadora: Roseli Saraiva Moreira Bittar.

Descritores: 1.Doenças vestibulares 2.Implante coclear 3.Vertigem 4.Surdez pós-lingual 5.Eletronistagmografia
\end{abstract}

\title{
USP/FM/DBD-155/14
}


"Eu não tenho paredes. Só tenho horizontes!!"

Mário Quintana 


\section{AGRADECIMENTOS}

\section{Agradeço}

Ao Prof. Dr. Ricardo Ferreira Bento, professor titular do Departamento de Otorrinolaringologia do HCFMUSP, pela oportunidade de poder realizar essa pesquisa.

À minha orientadora Prof. Dra. Roseli Saraiva Moreira Bittar que considero uma verdadeira mentora e um exemplo de profissionalismo. Além disso, possui a admirável capacidade de transformar a complexidade em simplicidade e tem o dom de indicar a pessoa certa para o trabalho certo. Foi um privilégio ter tido a oportunidade de estar ao seu lado e aprender sobre esse universo maravilhoso da Otoneurologia durante todos esses anos.

Ao meu querido Prof. Riquelme Romero Leal Portela que com muito carinho, me ensinou os primeiros princípios em otorrinolaringologia e que sempre teve muita confiança de que eu iria muito longe nesse caminho do saber.

Ao mestre Dr. Plínio Conceição dos Santos pelos seus ensinamentos em anatomia e fisiologia em otorrinolaringologia durante a residência na PUC de Sorocaba. Sempre me incentivando a fazer pesquisa e, sobretudo, a ingressar na vida acadêmica.

Ao Dr. Marco Aurélio Bottino, um verdadeiro pai, com quem aprendi mais do que do que otoneurologia. Sempre me incentivando com carinho e amor a seguir em frente.

Ao Dr. Luiz Ubirajara Sennes, pelo apoio e coordenação da Pósgraduação.

Ao amigo, vizinho e colega de trabalho Dr. Robinson Koji Tsuji, por ter contribuído com a minha pesquisa e facilitado meu trabalho. E, especialmente, por ter me dado mais que um voto de confiança.

Ao Dr. Mário EdvinGreters, que desde o início acompanhou minha pesquisa. Um verdadeiro amigo. Sempre com muita paciência, carinho e 
atenção guiou meus passos até aqui. Contribuiu muito para que minha pesquisa pudesse chegar até o fim.

Aos Pacientes, colaboradores desta pesquisa, e fonte de aprendizado, minha eterna gratidão.

Aos membros da banca de qualificação: Dra. Signe Schuster Grazel, Dr. Robinson Koji Tsuji e Dra. Jeanne Oiticica Ramalho pelas sugestões quanto à correção e aprimoramento desta pesquisa.

Ao Prof. Dr. Raymundo Soares, pela preciosa ajuda com a análise estatística dessa pesquisa.

À Dra. Camila de Giácomo Carneiro Barros, Dr. Ítalo Roberto Torres de Medeiros, Dra. Lucinda Simoceli e Dra. Maruska D’Aparecida Santos que admiro muitíssimo e que marcaram presença nessa minha jornada.

Às minhas amigas queridas, sócias, mães e mulheres Eliane Von Sohsten e Sandra Bastos, por podermos sempre compartilhar uma amizade leal, torcermos umas pelas outras e, o mais importante, estarmos sempre juntas.

À amiga Juliana Anauate pelo carinho e amizade.

À querida amiga Aline Bittencourt, que tanto me ajudou com dicas preciosas.

À amiga, vizinha e sócia Tatiane Vaccaro Campos, pelo apoio e torcida.

À amiga e fonoaudióloga Maysa Ubrig, com quem dividi pacientes, risos e lágrimas. Uma das poucas pessoas que viveu e sentiu na pele as dificuldades de realizar uma pesquisa dessa envergadura.

À amiga e irmã Fabiane, por sempre me ouvir e apoiar. Nossa amizade é atemporal.

À amiga, fonoaudióloga, vizinha e parceira de trabalho Cibele Brugnera, que participou ativamente da minha pesquisa, na interpretação dos dados das provas calóricas, e por quem tenho muito carinho, respeito e admiração. Um exemplo de amor e compaixão pelo próximo. 
Aos demais colegas do setor de Otoneurologia, Dr. Arlindo C. Lima Neto, Dra. Raquel Mezzalira, felows e residentes do Ambulatório de Otoneurologia do HCFMUSP, pela convivência harmônica durante estes anos.

À Maria Márcia Alves, Maria Marilede Alves, Lucivânia da Silva Quintão e Milva T. Luciano Braz, funcionárias do Departamento de Otorrinolaringologia do HCFMUSP, pela amizade, carinho e colaboração nos aspectos burocráticos e assistenciais na execução desta pesquisa.

Aos meus amados pais tortos Eliana e Antonio José Abramides, pelo interesse genuíno em tudo que eu faço e pelo apoio e amor incondicional.

À Cecília e Paulo Toledo, pelo interesse e carinho.

Ao meu pai Norberto Arena com quem aprendi o quão importante é ser apaixonado pelo que se faz.

À minha mãe Margareth por ter me ensinado o verdadeiro sentido da palavra que rege minha vida hoje e sempre: ORGANIZAÇÃO.

À minha tia Terezinha que sozinha vale por um fã-clube inteiro.

Às minhas queridas irmãs Deborah e Cintia que representam o feminino na minha vida.

Ao meu marido Vinícius, que é tudo para mim. Eu simplesmente não existo sem ele.

Às minhas filhas amadas Julia e Luiza, que são dois sóis que iluminam e aquecem minha vida e fazem tudo ter sentido.

À minha querida cunhada-irmã Cristina, que sempre esteve comigo em todos os momentos com amor sincero, amizade cristalina e companheirismo sem fim.

Aos sobrinhos Amanda, Helena e Teodoro por serem fonte sem fim de risos e alegria.

À querida Fernanda, fiel escudeira, que sempre cuidou com muito carinho de tudo na minha casa para que eu pudesse me dedicar ao meu trabalho. 


\section{SUMÁRIO}

Listas de Figuras e Gráficos

Lista de Tabelas

Listas de Abreviaturas

Listas de Siglas

Resumo

Abstract

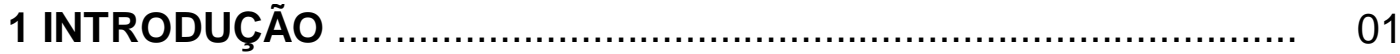

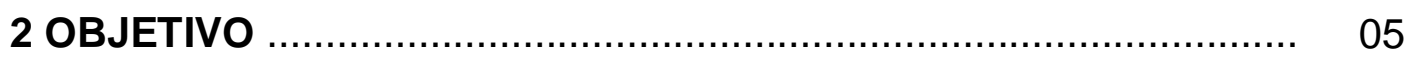

3 REVISÃO DE LITERATURA ............................................ 07

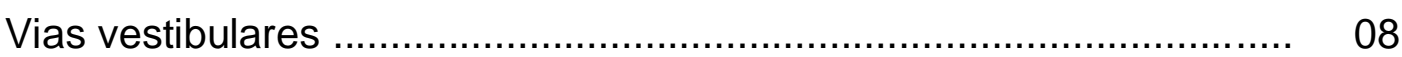

3.1 Implante coclear e função vestibular ....................................... 09

3.1.1 Efeito negativo do implante coclear sobre a função vestibular .... 11

3.1.2 Efeito positivo do implante coclear sobre a função vestibular..... 14

3.1.3 Efeito nulo do implante coclear sobre a função vestibular........... 15

3.2 Avaliação vestibular no implante coclear ................................. 16

4 CASUÍSTICA E MÉTODO ................................................. 20

4.1 Casuística ..................................................................... 21

4.1.1 Seleção da amostra ...................................................... 21

4.2 Metodologia ...................................................................... 22

4.2.1 Avaliação do equilíbrio corporal ............................................. 22

4.2.1.1 Anamnese ....................................................................... 25

4.2.1.2 Eletronistagmografia computadorizada ............................. 25

4.2.1.3 Prova rotatória pendular decrescente ................................. 25

4.2.1.4 Posturografia dinâmica computadorizada ........................... 26

4.3 Estatística ................................................................... 28

5 RESULTADOS ..................................................................... 30

5.1 Avaliação clínica ................................................................... 32

5.1 .1 Zumbido ....................................................................... 32

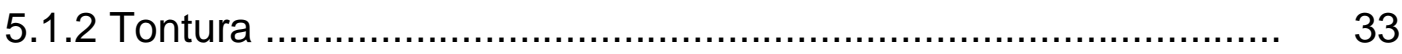




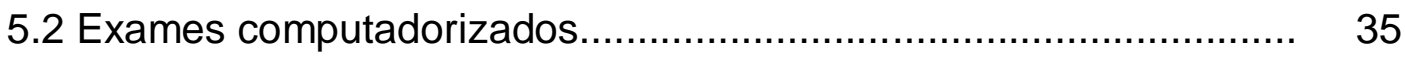

5.2.1 Prova rotatória pendular decrescente........................................ $\quad 35$

5.2.2 Eletronistagmografia............................................................. 36

5.2.3 Posturografia dinâmica computadorizada ................................ 37

5.2.4 Posturografia dinâmica computadorizada vs. prova calórica ....... 39

5.2.5 Comparação entre os grupos com resposta positiva e negativa. $\quad 39$

5.2.6 Evolução dos grupos com resposta positiva e negativa .............. 40

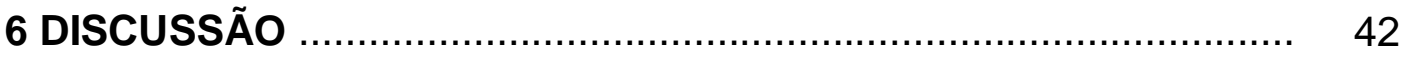

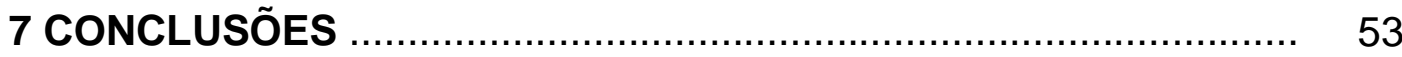

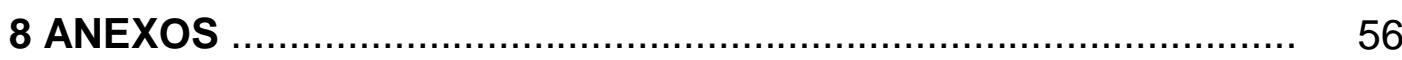

Anexo A - Anamnese ................................................................. 57

Anexo B - Causas, características e ocorrências de tontura em todos os momentos de avaliação ............................................ 60

Anexo C - Respostas das provas calóricas nas orelhas implantadas e não implantadas no Dia 0 e no dia 365 ............................ 61

Anexo D - Valores de $p$ nas diferentes condições de conflito sensorial, C1 a C6, do Índice final de equilíbrio, calculados por meio do teste de Wilcoxon ........................................... 62

Anexo E-Valores de $p$ nas diferentes condições de conflito sensorial, C1 a C6, e para o Índice final de equilíbrio, em grupos respostas negativa e/ou positiva para PDC nos Dias 0 e/ou 365 ............................................................ 65

9 REFERÊNCIAS

APÊNDICES

Apêndice 1 - Carta da Comissão de Ética

Apêndice 2 - Termo de Consentimento Livre e Esclarecido 


\section{LISTAS DE FIGURAS E GRÁFICOS}

Figura 1 Fluxograma do estudo

Figura 2 Condições de estimulação sensorial no Teste de Integração Sensorial

Gráfico 1 Ocorrência de zumbido em todos os momentos de avaliação e significância $(p)$ da diminuição do evento em relação à avaliação pré IC

Gráfico 2 Ocorrência de tontura em todos os momentos de avaliação e significância $(p)$ da diminuição do evento em relação à avaliação pré IC 


\section{LISTA DE TABELAS}

Tabela 1 Causas da surdez dos sujeitos submetidos ao IC na amostra avaliada

Tabela 2 Avaliação da função vestibular em orelha implantada e orelha não implantada no Dia 365 ao comparar com o Dia 0

Tabela 3 Valor de $p$ nas Condições 1 a 6 da PDC nos Dias 60, 120, 180 e 365 em relação ao Dia 0

Tabela 4 Valor de $p$ na comparação entre pacientes portadores de arreflexia pós-calórica e pacientes com resposta na PC nos Dias 0 e 365 


\section{LISTA DE SIGLAS}

CAPPesq Comissão de Ética para Análise de Projetos de Pesquisa FMUSP Faculdade de Medicina da Universidade de São Paulo HC Hospital das Clínicas 


\section{LISTAS DE ABREVIATURAS}

$\begin{array}{ll}\text { AASI } & \text { Aparelho de Amplificação Sonora Individual } \\ \text { AVB } & \text { Arreflexia Vestibular Bilateral } \\ \text { AVC } & \text { Acidente Vascular Cerebral } \\ \text { CR } & \text { Cadeira Rotatória } \\ \text { Dia 0 } & \text { dia que antecedeu a cirurgia de implante coclear } \\ \text { Dia 1 } & \text { dia em que foi realizada a cirurgia de implante coclear } \\ \text { Dia 30 } & \text { dia da ativação do implante coclear 30 dias após a cirurgia } \\ \text { Dia 60 } & \text { 1' retorno 60 dias após a cirurgia de implante coclear }^{\circ} \\ \text { Dia 120 } & 2^{\circ} \text { retorno 120 dias após a cirurgia de implante coclear } \\ \text { Dia 180 } & 3^{\circ} \text { retorno 180 dias após a cirurgia de implante coclear } \\ \text { Dia 365 } & 4^{\circ} \text { retorno 365 dias ou 1ano após a cirurgia de implante coclear } \\ \text { et al. } & \text { e outros } \\ \text { ENG } & \text { Eletronistagmografia } \\ \text { IC } & \text { Implante Coclear } \\ \text { IE } & \text { Índice Final de Equilíbrio } \\ \text { OI } & \text { Orelha Interna } \\ \text { OTN } & \text { Otoneurológico } \\ \text { PDC } & \text { Posturografia Dinâmica Computadorizada } \\ \text { PRPD } & \text { Prova Rotatória Pendular Decrescente } \\ \text { RVE } & \text { Reflexo Vestíbulo Espinal } \\ \text { RVO } & \text { Reflexo Vestíbulo Ocular } \\ \text { SNC } & \text { Sistema Nervoso Central } \\ \text { TCE } & \text { Traumatismo Crânio Encefálico } \\ \text { TIS } & \text { Teste de Integração Sensorial } \\ \text { VACL } & \text { Velocidade Angular da Componente Lenta } \\ \text { VNG } & \text { Videoeletronistagmografia } \\ \text { VPPB } & \text { Velocidade Postural Paroxística Benigna } \\ \text { F } & \text { feminino } \\ & \end{array}$


masculino

nl normorreflexia

tontura

Z zumbido

0 arreflexia 


\section{LISTAS DE SÍMBOLOS}

$\begin{array}{ll}\text { o } & \text { grau } \\ + & \text { presente } \\ - & \text { ausente } \\ * & \text { valor apresenta significância estatística } \\ \downarrow & \text { hiporrefelexia } \\ \uparrow & \text { hiperrelflexia } \\ \% & \text { porcento } \\ = & \text { igual }\end{array}$




\section{RESUMO}

Abramides PA. Avaliação sequencial do equilíbrio pré e pós-implante coclear em pacientes com surdez pós-lingual [tese]. São Paulo: Faculdade de Medicina, Universidade de São Paulo; 2014.

INTRODUÇÃO: A literatura é discordante com relação à interferência do IC sobre o equilíbrio corporal. Sendo assim, resolvemos avaliar o equilíbrio corporal de pacientes surdos pós-linguais, submetidos a implante coclear unilateral. OBJETIVO: Observar o equilíbrio corporal pré e pós-implante coclear (IC) ao longo de 1 ano. CASUÍSTICA E METODOLOGIA: Estudo prospectivo observacional realizado com 24 pacientes adultos, surdos pós-linguais submetidos à avaliação vestibular antes e depois da cirurgia de implante coclear unilateral. A avaliação vestibular contou com um questionário sobre vertigem, prova calórica (PC), cadeira rotatória (CR) e posturografia dinâmica computadorizada (PDC) aplicados no pré-operatório, 60, 120, 180 dias e 1 ano após a cirurgia de IC. RESULTADOS: A tontura foi referida por 13 (54,2\%) pacientes pré-IC, enquanto $11(45.8 \%)$ não apresentaram a queixa. Ao final do estudo 11 sujeitos $(84,6 \%)$ referiram melhora da tontura, em $1 \quad(7,7 \%)$ permaneceu inalterada e em $1(7,7 \%)$ piorou. Dos 24 pacientes apenas 5 indivíduos $(20,8 \%)$ desenvolveram tontura no pós-operatório imediato com resolução completa após um mês. A prova calórica identificou 7 (29,2\%) sujeitos normorreflexos, 8 (33,3\%) com hiporreflexia ou arreflexia unilateral , 3 $(12,5 \%)$ com hiporreflexia bilateral e 6 (25\%) com arreflexia vestibular bilateral (AVB).Houve interferência do estímulo elétrico em ambas as orelhas e na evolução da recuperação postural após ativação do IC, que promoveu a melhora significativa dos índices da PDC ao longo de um ano de acompanhamento. Ao final do estudo, as médias numéricas das condições avaliadas pela PDC mostraram-se superiores nos indivíduos que apresentaram resposta à prova calórica em relação àqueles que possuíam AVB. CONCLUSÃO: Foi decisiva a presença ou não de resposta pós-calórica na evolução do equilíbrio corporal ao longo de 1 ano. A ausência de resposta póscalórica na avaliação pré-operatória implicou em pior prognóstico na evolução do equilíbrio corporal. No entanto, o melhor desempenho postural dos sujeitos com AVB pode ser explicado pelo melhor aproveitamento da informação visual. É fundamental documentar a presença de função vestibular antes da cirurgia de IC, pois dela depende o prognóstico do individuo em relação às habilidades de aprendizado e recuperação postural ao longo do tempo.

Descritores: 1.doenças vestibulares 2.implante coclear 3.vertigem 4.surdez pós-lingual, 5.eletronistagmografia 


\begin{abstract}
Abramides PA. Sequential study of vestibular function pre- and post cochlear implantation in postlingual deafened patients [thesis]. São Paulo: "Faculdade de Medicina, Universidade de São Paulo"; 2014.
\end{abstract}

INTRODUCTION: There is no consensus in the literature with regard to the effects of cochlear implantation $(\mathrm{Cl})$ on vestibular function and balance in patients with deafness. Because of this fact we decided to assess vestibular function before and after unilateral cochlear implantation $(\mathrm{Cl})$ in patients with postlingual deafness. OBJECTIVE: To assess balance before and after cochlear implantation $(\mathrm{Cl})$ over the course of 1 year. PATIENTS AND METHODS: prospective, observational study sought to assess balance in 24 postlingually deaf adults undergoing vestibular evaluation before and after cochlear implantation $(\mathrm{Cl})$. Vestibular assessment consisting of a vertigo questionnaire, caloric tests (CT), rotary chair testing ( $\mathrm{RC}$ ), and computerized dynamic posturography (CDP) was performed preoperatively and at 60,120, 180 days and 1 year after $\mathrm{Cl}$. RESULTS: Overall, 13 patients (54.2\%) reported preoperative dizziness and $11(45.8 \%)$ did not have the symptom pre-Cl. At the end of the study dizziness ameliorated in $11(84.6 \%)$, remained unchanged in 1 $(7.7 \%)$ and worsened in $1(7.7 \%)$. Only 5 of the 24 patients $(20.8 \%)$ developed immediate postoperative dizziness, which resolved within a month. The caloric tests identified $7(29.2 \%)$ patients with normal reflexes, 8 (33.3\%) with unilateral areflexia or hyporeflexia, $3(12.5 \%)$ with bilateral hyporeflexia, and $6(25 \%)$ with bilateral vestibular loss (BVL). Electrical stimulation affected both ears and interfered with the progression of postural recovery after $\mathrm{Cl}$ activation, which led to a significant improvement in CDP values over the course of 1 year of followup. At the end of the study, the mean values of the conditions assessed by CDP were higher in individuals who had responded to caloric tests than in individuals with BVL. The better postural performance of subjects with BVL may be due to better use of visual information. CONCLUSION: The presence or absence of CT response was a decisive determinant of balance outcomes over the year after surgery. The absence of post-caloric response in preoperative assessment resulted in a worse prognosis in the evolution of body balance. However, patients with BVL were able to use the visual information for postural stabilization with improvement in the Composite Score. It is essential that vestibular assessment findings be documented before $\mathrm{Cl}$ surgery because a patient's prognosis in terms of learning skills and postural recovery over time depends on this information.

Descriptors: 1.vestibular diseases 2.cochlear implant 3.vertigo 4.postlingual deafness 5.eletronystagmography 
1 INTRODUÇÃO 


\section{INTRODUÇÃO}

Ouvir é uma função que nos auxilia a compreender o mundo ao nosso redor. Foi com a finalidade de resgatar essa função que vários pesquisadores se dedicaram ao desenvolvimento de alguma interface que pudesse restituir a percepção do som. A partir dessas iniciativas, foi desenvolvido o implante coclear (IC), uma opção terapêutica para pacientes com surdez neurossensorial profunda (Oliveira, Bernal, 2002).

O interesse pela função vestibular, em pacientes portadores de IC, teve início, quando alguns pacientes implantados evoluíram com tontura no pós-operatório. Desde que Black (1977b) e Black et al. (1980) demonstraram redução ou ausência das respostas nas provas calóricas de pacientes submetidos ao IC, muitos outros estudos relataram a possibilidade do IC ocasionar uma disfunção vestibular (Huygen et al., 1995; Pierchala, 1996; Ito, 1998; Bouccara et al., 2005; Enticott et al., 2006). De fato, as opiniões dos autores são controversas. Alguns acreditam que o IC interfere negativamente na função vestibular (Huygen et al., 1995; Brey et al., 1995; Vibert et al., 2001; Steenerson et al., 2001; Enticott et al., 2006), enquanto outros acreditam que o IC não apresenta qualquer efeito nesse sentido (Eisenberg et al., 1982; Migliaccio et al., 2005; Suarez et al., 2007). Há, ainda, autores que acreditam que o IC pode melhorar o equilíbrio corporal (Bance et al., 1998; Szirmai et al., 2001; Ribari et al., 2002; Buchman et al., 2004). 
Sabe-se da existência de conexões vestibulococleares, confirmadas por meio de exames histológicos (Rasmussen, 1940; Arnesen, 1984), dissecções de ossos temporais (Silverstein, 1984; Nageris et al., 2000), estudos eletrofisiológicos (Burian et al., 1989), técnicas radiológicas (Kim et al., 1998), e microscopia eletrônica (Özdogmus et al., 2004). Existem evidências clínicas da interferência do IC na função vestibular. Uma das descrições de literatura mais interessantes relata a melhora da função contralateral ao IC. Alguns pacientes implantados apresentaram melhora das respostas pós-calóricas da outra orelha, o que sugere uma atividade benéfica por atuação da plasticidade neuronal (Ribari et al., 2002). Ainda sem uma explicação definitiva, supõe-se que os estímulos aferentes seriam capazes de induzir melhora funcional do sistema vestibular (Enticott et al., 2006). Enfim, os mecanismos que levam à melhora da função vestibular contralateral não são claros (Osberger et al., 1998; Lohle et al., 1999).

Os efeitos do implante coclear sobre o equilíbrio corporal despertou, em nossa prática clínica, a necessidade de desenvolver um protocolo para avaliação mais adequada, uma vez que não há consenso na literatura a respeito do tema.

Acreditamos que a investigação da função vestibular, em pacientes surdos pré IC estima o prognóstico do indivíduo em relação às habilidades de aprendizado e recuperação postural ao longo do tempo. Seria possível, ainda, nortear a escolha do lado a ser implantado e oferecer ao paciente a escolha entre audição e equilíbrio, se for o caso. 
Com base nos fatos expostos, preparamos um projeto de pesquisa que tem a finalidade de avaliar o equilíbrio corporal, imediatamente antes e após a cirurgia do IC. Nossa intenção é observar a evolução da função vestibular em longo prazo, por meio de interrogatório clínico e medidas objetivas, para estabelecer a melhor forma de avaliação e seguimento de pacientes implantados. 
2 OBJETIVO 


\section{OBJETIVO}

Avaliar sequencialmente o equilíbrio de pacientes portadores de perda auditiva neurossensorial profunda bilateral pós-lingual submetidos à cirurgia de implante coclear ao longo de um ano, para responder as questões seguintes:

1. Presença e evolução da tontura e do zumbido antes e após o IC;

2. Influência da presença de função vestibular pré-operatória na evolução do equilíbrio; e,

3. Melhora da postura por meio da posturografia dinâmica computadorizada (PDC). 
3 REVISÃO DE LITERATURA 


\section{REVISÃO DE LITERATURA}

O IC tem reabilitado pacientes surdos ao longo dos últimos 20 anos. É indicado para pacientes portadores de deficiência auditiva neurossensorial bilateral de grau severo a profundo, que não se beneficiam com o uso de aparelho de amplificação sonora individual (AASI). Esses indivíduos apresentam escores inferiores a $50 \%$, em testes de reconhecimento de sentenças com o uso da melhor protetização bilateral possível. Com a evolução da técnica cirúrgica, hoje o implante coclear tem sido indicado também para: crianças, pacientes com audição residual preservada, surdez unilateral e zumbido incapacitante. Pode ser realizado bilateralmente e, às vezes, simultaneamente (Krause et al., 2009d).

\section{Vias vestibulares}

Os núcleos vestibulares integram sinais provenientes dos órgãos vestibulares àqueles oriundos da medula espinal, cerebelo e sistema visual, direcionando-os para vários outros pontos do sistema nervoso central (SNC): núcleos oculomotores, formação reticular, centros medulares, cerebelo e tálamo. Os sinais são integrados entre si e originam comandos eferentes, que geram o reflexo vestíbulo ocular (RVO) e o reflexo vestíbulo espinal (RVE) nos órgãos efetores periféricos (Kandel et al., 2003; Herdman, 2007). Há, ainda, vias neurais que conectam um núcleo vestibular ao núcleo 
contralateral (Kandel et al., 2003) por fibras intercomissurais, que permitem o compartilhamento de informações. Por meio das vias vestibulares e suas conexões, a lesão de estruturas periféricas pode ser compensada por substituições sensoriais e adaptações neurais, processo denominado compensação vestibular ou compensação central (Baloh, Honrubia, 2001).

Além disso, devemos destacar que as vias cocleares apresentam pontos de interação com o sistema vestibular, comprovada por exames histológicos (Rasmussen, 1940; Arnesen, 1984), dissecções de ossos temporais (Silverstein, 1984; Nageris et al., 2000), estudos eletrofisiológicos (Burian et al., 1989), técnicas radiológicas (Kim et al., 1998) e microscopia eletrônica (Özdogmus et al., 2004).

\subsection{Implante coclear e Função vestibular}

Não há evidência de que a corrente elétrica produzida pelo IC possa alterar o equilíbrio. No entanto, em pacientes que apresentam perda total ou parcial da função vestibular, além da surdez profunda, parece que sua estabilidade postural após ativação do IC melhora (Eisenberg et al., 1982).

São propostos alguns mecanismos como possíveis causadores da vertigem pós-operatória. São eles: o trauma durante a inserção do eletrodo, a perda de perilinfa intra-operatória, a reação por corpo estranho, a fístula perilinfática, a hidropisia endolinfática traumática, a estimulação elétrica 
vestibular pelo implante e a idade avançada (Cohen et al., 1991; Bance et al., 1998; Kubo et al., 2001; Fina et al., 2003).

O tipo de técnica empregada para inserção do IC é outro fator que apresenta impacto sobre a função vestibular. Quando o IC é colocado na escala timpânica através da janela redonda, há um risco menor de perda de função vestibular e vertigem (Tien et al., 2002; Todt et al., 2008).

O trauma em razão da inserção do eletrodo pode causar mudanças morfológicas e funcionais na orelha interna. Estudos histológicos sobre o osso temporal têm demonstrado que, tanto a cóclea quanto os órgãos vestibulares periféricos, são afetados (Fayad et al., 2006). O sáculo é o local mais afetado, seguido pelo utrículo e canais semicirculares (Tien et al., 2002; Handzel et al., 2005), com prejuízo funcional dos receptores vestibulares periféricos (Steenerson et al., 2001; Vibert et al., 2001; Enticott et al., 2006; Todt et al., 2008; Krause et al., 2010). Apesar das descrições histológicas o comprometimento tissular pode ser assintomático (Tien et al., 2002; Krause et al., 2009b).

O tipo de IC não apresenta relação com a ocorrência de problemas vestibulares no pós-operatório (Todt et al., 2008).

É documentado, ainda, que o tipo de alteração funcional causado pelo IC é balizado também por fatores anatômicos, pela predisposição individual ao padrão de estímulo produzido pelo IC e, também, pela capacidade plástica do sistema neural de cada indivíduo (Abramides et al., 2009). 
Os indivíduos surdos apresentam substituição cross-modal do córtex auditivo por representação visual, condição que determina o prognóstico auditivo do paciente que receberá o IC (Lomber et al., 2010; Lee et al., 2001). Dessa maneira, a experiência auditiva, tanto a privação quanto a estimulação gerada pelo IC, podem afetar ambos os sistemas, auditivo e visual, nesses pacientes (Doucet et al., 2006; Buckley et al., 2011).

3.1.1 Efeito negativo do IC sobre a função vestibular

Muitos autores acreditam que o IC apresente influência negativa sobre a função vestibular (Black, 1977a,b; Black et al., 1987).

Em estudo realizado por Enticott et al. (2006) com 146 pacientes com idades entre 20 e 90 anos, foi constatado que 32\% evoluíram com algum distúrbio do equilíbrio após a cirurgia de IC unilateral e resposta reduzida na eletronistagmografia (ENG). Os autores também verificaram que pacientes com 70 anos ou mais apresentaram respostas bastante diminuídas na ENG.

Para Huygen et al. (1995), o risco de perda de função vestibular foi estimado em 31\%. Já para Melvin et al. (2008) o risco de perda de função vestibular secundária ao IC unilateral, não difere de suas outras complicações como meningite pós IC e lesão do nervo facial, e gira em torno de $0,2 \%$ a $0,4 \%$.

Ito (1998) ao analisar 55 pacientes implantados após um mês do procedimento descreve deterioração da função vestibular em 38\% dos 
casos, confirmada por prova calórica. Vinte e seis (47\%) dos 55 pacientes apresentaram tontura, desses, em 15 (58\%) indivíduos o sintoma ocorreu 15 dias após o IC. Em nove (34\%), a tontura persistiu por mais de duas semanas e em dois pacientes (8\%) ocorreu um mês após o IC.

Filipo et al. (2006) avaliaram 93 pacientes por meio de videoeletronistagmografia (VNG), plataforma de força e cadeira rotatória cinco semanas após a cirurgia de IC, e ainda 30, 60 e 90 dias após sua ativação. Constataram que: tanto o IC quanto sua ativação podem comprometer o sistema vestibular no pós-operatório imediato. Já para Magnusson et al. (1995), pacientes com surdez pós-lingual tem controle postural reduzido e são mais sensíveis à estimulação elétrica do nervo vestibular.

Bouccara et al. (2005) encontraram nove crianças (3\%) e 17 adultos (16\%) com problemas vestibulares entre os 469 pacientes implantados. Entre as crianças, $50 \%$ tiveram vertigem transitória no pós-operatório imediato, $25 \%$ vertigem persistente nos três primeiros meses e $25 \%$ após três meses. Entre os adultos $52 \%$ apresentaram vertigem pós-operatória, $28 \%$ nos três primeiros meses e $20 \%$ três meses após o IC. Portanto, é de suma importância informar ao paciente sobre o risco de sintomas vestibulares causados pelo IC no pós-operatório A maioria dos sintomas costuma ser transitória, mas quando persistente a reabilitação vestibular pode beneficiar esses pacientes (Brey et al., 1995). 
Em relação à função otolítica e canalicular, Vibert et al. (2001) constataram que a função canalicular pode ficar prejudicada pós IC em $20 \%$ dos casos e a função otolítica, tanto do lado implantado quanto do contralateral, mantém-se preservada.

Para Fina et al. (2003), a tontura poderia resultar de uma hidropisia endolinfática em consequência de mudanças crônicas na orelha interna $(\mathrm{OI})$. Em estudo feito pelos autores, 39\% dos pacientes implantados evoluíram com tontura tardia. Kubo et al. (2001) também acreditam que as lesões da Ol secundárias ao IC desenvolvem-se gradualmente e os fatores clínicos que as desencadeiam são os mesmos que os da doença de Ménière.

Existe, ainda, a hipótese de que a vertigem postural paroxística benigna (VPPB) possa ser uma sequela da cirurgia de IC (Limb et al., 2005). Dois mecanismos explicariam a doença: inclusão de partículas de pó de osso no interior da cóclea durante a cocleostomia, e deslocamento dos otólitos, causado pelo broqueamento da cóclea e/ou por estimulação elétrica (Limb et al., 2005; Viccaro et al., 2007). A VPPB pode ocorrer na orelha implantada sem relação cronológica com o procedimento cirúrgico ou a ativação IC e não influencia a percepção de fala dos pacientes (Zanetti et al., 2007). Quanto ao seu diagnóstico, tratamento e prognóstico, não há diferença de conduta entre implantados e não implantados (Limb et al., 2005) 


\subsubsection{Efeito positivo do IC sobre a função vestibular}

Buchman et al. (2004) verificaram melhora significativa na medida objetiva da estabilidade postural em pacientes submetidos a implante coclear unilateral utilizando posturografia dinâmica computadorizada (PDC).

Em 2002, Ribari et al. notaram melhora da velocidade angular da componente lenta (VACL) do labirinto contralateral ao implante em $30 \%$ dos casos. Os autores consideram que a plasticidade das sinapses químicas, possa ser modificada funcional e anatomicamente durante a evolução pósoperatória por meio da experiência e do aprendizado. Os impulsos auditivos aferentes poderiam estimular o sistema vestibular, promovendo assim a melhora de suas respostas (Enticott et al., 2006). Os mecanismos envolvidos na melhora da função do labirinto contralateral ainda não são claros, mas a plasticidade do sistema neural pode explicá-los (Ribari et al., 1999; 2002).

Bonucci et al. (2008) avaliaram 38 pacientes no pré-operatório de IC e verificaram que 22 (58\%) apresentavam desequilíbrio. Entre os 22 pacientes 66\% apresentavam alteração da ENG e 34\% eram normorreflexos. Dos 38 pacientes, 31 foram avaliados no pós-operatório, 25 (81\%) evoluíram sem queixas imediatas e seis (19\%) apresentaram desequilíbrio tanto pré-operatório quanto no pós-operatório imediato e pósativação. No pós-operatório, 81\% tinham ENG alterada, 16\% normal e em $3 \%$ o resultado foi inconclusivo. A principal queixa foi tontura, seguida de vertigem postural e não postural, tanto no pré quanto no pós-operatório. No 
entanto, $17 \%$ deixaram de referir desequilíbrio e apenas 3\% tiveram piora pós-IC. Os autores atribuem a melhora observada à compensação vestibular ou à estimulação elétrica. IC.

Szirmai et al. (2001), ao compararem 60 provas calóricas a ar, no pré e pós IC, verificaram que 20 não apresentaram mudança da função vestibular, em 14 houve piora e em 16 houve melhora da função. Para os autores, o motivo da melhora da resposta vestibular não está claro.

3.1.3 Efeito nulo do IC sobre a função vestibular

Suarez et al. (2007) realizaram ENG e posturografia em crianças implantadas, com idades entre 8 e 11 anos, e concluíram que, apesar da função vestibular deficiente, crianças surdas conseguem apresentar controle de equilíbrio adequado e que o IC não afeta a estratégia de organização sensorial.

Para Himi et al. (1995), não houve mudança dos resultados pré e pósoperatórios em relação à plataforma de força e o dano vestibular após a cirurgia IC foi considerado mínimo. A vertigem foi passageira no pósoperatório imediato e ocorreu em cinco dos 18 pacientes (28\%). Concluíram que o resultado do exame vestibular no pré-operatório pode ser útil como fator preditivo no pós-operatório.

Por sua vez, Ito (1998) encontrou 63\% de pacientes normorreflexos no pós-operatório e acredita que se o IC afeta a função vestibular ela é 
rapidamente compensada.

Segundo Migliaccio et al. (2005), o RVO não mudou após IC em 10 dos 11 pacientes testados pré IC. Apenas um paciente evoluiu com perda parcial de função na orelha implantada, e foi o único que apresentou vertigem passageira e oscilopsia pós IC. Os autores concluíram que as disfunções vestibulares no pré-operatório foram comuns entre os pacientes candidatos ao IC e a lesão em sequência ao IC foi incomum.

De acordo com Eisenberg et al. (1982), o IC não alterou o equilíbrio em grau significante em pacientes adultos com surdez profunda.

O comprometimento da função vestibular no pré-operatório é comum entre os pacientes candidatos ao IC, o que não ocorre no pós-operatório (Migliaccio et al., 2005).

\subsection{Avaliação vestibular no Implante coclear}

O exame vestibular pode ser proposto como um exame adicional para a avaliação dos candidatos ao IC unilateral (Filipo et al., 2006). Ele permite pesquisar disfunções uni ou bilaterais, evitando a iatrogênia e a arreflexia bilateral pós-operatória, avaliar o nível de compensação e auxiliar na escolha do lado a ser implantado (Bouccara et al., 2005; Huygen et al., 1995). De acordo com Jacot et al. (2009), a arreflexia pós-operatória é estimada em 
$10 \%$ dos casos e a avaliação vestibular sistemática pré e pós IC seria capaz de fornecer uma taxa mais precisa, especialmente em crianças.

Segundo Rossi et al. (1998), quando não existe preferência quanto ao lado a ser implantado, deve-se levar em conta a avaliação vestibular. Visto que, quando a orelha a ser implantada é a do labirinto funcionante e a outra orelha apresenta uma arreflexia unilateral, existe o risco de arreflexia bilateral pós IC. Nesse caso, é melhor implantar o lado arreflexo para evitar risco de arreflexia bilateral (Huygen et al., 1994).

Em estudo feito por Chiong et al. (1994), a resposta da ENG préoperatória permitiu definir o lado do implante. O IC foi colocado no lado com menor resposta, reduzindo, assim, a chance de prejuízo da função vestibular residual. Dos 16 pacientes que possuíam função vestibular residual, apenas três $(19 \%)$ apresentaram redução das respostas na ENG pós IC. Em nenhum paciente ocorreu perda total da função vestibular pós IC. Os autores concluíram que lesões vestibulares pós IC são incomuns.

Segundo a American Academy of Neurology Technology Assessment, as provas calóricas continuam sendo a técnica adequada, amplamente aceita e útil na detecção de distúrbios vestibulares, especialmente nos comprometimentos unilaterais. Já a prova rotatória é considerada o método gold standard na documentação da perda vestibular bilateral. (Goebel et al., 1995; Furman et al., 1996)

Segundo Szirmai et al. (2001), a prova calórica a ar é um método seguro e útil na avaliação das mudanças do sistema vestibular no pós- 
operatório de IC. No entanto, para Bance et al. (1998) a prova calórica não é um exame adequado para avaliar função vestibular residual, porque o estímulo para o canal lateral é de frequência baixa, e pode resultar em hiporreflexia, mesmo na presença de resposta às frequências altas principal função das cristas ampolares. Para tanto, a avaliação deve ser complementada com a prova rotatória considerada gold standard no diagnóstico de arreflexia vestibular (Bance et al., 1998). Mangham (1987) observou que nove de seus 12 pacientes candidatos ao IC apresentaram resposta na prova calórica. Quatro deles sofreram redução do RVO e desequilíbrio leve por mais de uma semana pós-cirurgia de IC. Nenhum paciente apresentou redução do RVO ou sintomas vestibulares durante a estimulação elétrica em seu estudo. Para Mangham (1987) isso indica que o exame é apropriado para monitorar a função vestibular em alguns implantados, mas não em todos.

Graças ao ENG e o questionário desenvolvido especificamente para avaliar desequilíbrio em implantados, Krause et al. (2008; 2009c) observam que em pacientes que evoluem com vertigem no pré-operatório, o exame vestibular é alterado com maior frequência que em pacientes sem vertigem. Para os autores, quando estruturas vestibulares são lesionadas durante 0 IC, esses pacientes desenvolvem sintomas relevantes, pois já possuem mecanismo de compensação central prejudicado, problemas proprioceptivos e/ou do RVO. Para entendermos os motivos que levam os pacientes implantados a desenvolver vertigem no pós-operatório é necessária uma análise vestibular prospectiva e acompanhamento dos sintomas. 
A PDC permite melhorar o índice diagnóstico de pacientes portadores de distúrbios de equilíbrio; localizar precocemente o sistema responsável pelo desequilíbrio com sensibilidade e especificidade; documentar e monitorizar o tratamento dos pacientes portadores de distúrbios do equilíbrio corporal, especialmente em crianças (Suarez et al., 2007).

Não há consenso na literatura sobre qual é o melhor método de avaliação vestibular pré e pós IC, fato que não descarta sua realização (Buchman et al., 2004). 
4 CASUÍSTICA E MÉTODO 


\section{CASUÍSTICA E MÉTODO}

Este estudo configura um ensaio clínico prospectivo longitudinal.

O protocolo de pesquisa foi aprovado pela Comissão de Ética para Análise de Projetos de Pesquisa - CAPPesq da Diretoria Clínica do Hospital das Clínicas e da Faculdade de Medicina da Universidade de São Paulo (FMUSP) em sessão de 20.02.2008 sob o número 0983/07 (Apêndice 1).

Os pacientes que participaram da presente pesquisa assinaram Termo de Consentimento Livre e Esclarecido (Apêndice 2).

\subsection{Casuística}

Foram avaliados 24 pacientes, de ambos os gêneros, provenientes do Ambulatório de Implante Coclear do Departamento de Otorrinolaringologia do Hospital das Clínicas da FMUSP, entre fevereiro de 2008 e setembro de 2011.

4.1.1 Seleção da amostra

Para a admissão no estudo foram considerados os critérios seguintes: Critérios de inclusão:

- Pacientes portadores de surdez pós lingual; 
- Pacientes com deficiência auditiva neurossensorial bilateral de grau severo a profundo, sem benefício com o uso de AASI ou aqueles que apresentam índices inferiores a 50\% em testes de reconhecimento de sentenças com o uso da melhor protetização bilateral possível.

Critérios de exclusão:

- Pacientes com indicação de abordagem cirúrgica via fossa média;

- Pacientes com alterações ortopédicas, neurológicas ou visuais que pudessem interferir nos resultados da PDC.

\subsection{Metodologia}

Com a finalidade de avaliar a evolução do equilíbrio corporal no primeiro ano após a cirurgia de implante coclear, os pacientes selecionados foram submetidos à avaliação sequencial em cinco oportunidades, antes e após a cirurgia de implante coclear.

\subsubsection{Avaliação do equilíbrio corporal}

Ao considerar o dia da cirurgia de implante coclear o primeiro dia do estudo (Dia 1), a rotina de avaliação sequencial do equilíbrio corporal compreendeu cinco momentos distintos:

1. No dia que antecedeu a cirurgia de IC unilateral (Dia 0);

2. 60 dias após a cirurgia (Dia 60); 
3. 120 dias após a cirurgia (Dia 120);

4. 180 dias após a cirurgia (Dia 180);

5. 1 ano depois da cirurgia (Dia 365).

Cada uma das avaliações compreendeu:

1. Anamnese (Anexo A);

2. Eletronistagmografia (ENG);

3. Prova Rotatória Pendular Decrescente (PRPD);

4. Posturografia Dinâmica Computadorizada (PDC).

O fluxograma do estudo pode ser observado na Figura 1. 
DIA 0 avaliação clínica + ENG + PRPD + PDC

DIA 1 cirurgia de implante coclear

DIA 30 ativação do implante coclear

DIA 60 avaliação clínica + ENG + PRPD + PDC

DIA 120 avaliação clínica + ENG + PRPD + PDC

DIA 180 avaliação clínica + ENG + PRPD + PDC

DIA 365 avaliação clínica + ENG + PRPD + PDC

Figura 1 - Fluxograma do estudo. (ENG - Eletronistagmografia; PRPD - Prova Rotatória Pendular Decrescente; PDC - Posturografia Dinâmica Computadorizada) 


\subsubsection{Anamnese}

A caracterização clínica das alterações cócleo-vestibulares foi realizada por meio de um questionário aplicado a todos os pacientes (Anexo A). Os sintomas foram classificados de acordo com tipo, duração, intensidade e fatores associados. Foi também realizado o exame físico otorrinolaringológico completo.

\subsubsection{Eletronistagmografia computadorizada}

Para a realização da eletronistagmografia computadorizada (ENG) foi utilizado o equipamento Micromedical Technologies versão 8.1 (META-4, USA).

A rotina da investigação compreendeu a pesquisa da oculomotricidade, que inclui as sacadas, rastreio, nistagmo optocinético, nistagmo espontâneo, semi-espontâneo e as posicionais (decúbito lateral direita e esquerda, torcional direita e esquerda, e Rose). A prova calórica (PC) por estimulação com água foi feita em ambas as orelhas com água quente $\left(44^{\circ} \mathrm{C}\right)$ e fria $\left(30^{\circ} \mathrm{C}\right)$.

\subsubsection{Prova rotatória pendular decrescente}

Para realizar a prova rotatória pendular decrescente (PRPD), foi utilizada cadeira pendular rotatória microprocessada modelo CGM-4, versão 6.0 (SCV-6.0, Contronic, Brasil). A cadeira gira sob seu próprio eixo central, 
a partir de uma angulação de $90^{\circ}$. Nesta prova, o paciente encontra-se sentado com a cabeça inclinada para frente cerca de $30^{\circ}$, com os olhos fechados. É, então, submetido a uma aceleração angular progressivamente decrescente, enquanto realiza tarefas cognitivas a fim de evitar inibição cortical dos movimentos oculares.

\subsubsection{Posturografia dinâmica computadorizada}

O equipamento utilizado para o exame de posturografia dinâmica computadorizada foi o Equitest ${ }^{\circledR}$, marca NeuroCom International Inc (USA).

O protocolo escolhido para a avaliação na PDC foi o Teste de Integração Sensorial (TIS). O teste permite avaliar a participação dos diversos componentes do equilíbrio corporal e sua integração sensorial na resposta reflexa motora frente às diversas situações que desafiam a estabilidade corporal. Foram aplicadas seis condições de conflito sensorial, três vezes cada uma, durante vinte segundos.

Condição 1 (C1): paciente em posição ortostática, plataforma fixa e olhos abertos;

Condição 2 (C2): paciente em posição ortostática, plataforma fixa e olhos fechados;

Condição 3 (C3): paciente em posição ortostática, plataforma fixa, olhos abertos e campo visual móvel;

Condição 4 (C4): paciente em posição ortostática, plataforma instável, olhos abertos com campo visual fixo; 
Condição 5 (C5): paciente em posição ortostática, plataforma instável e olhos fechados;

Condição 6 (C6): paciente em posição ortostática, plataforma instável e campo visual em movimento, com os olhos abertos.

Índice Final de Equilíbrio (IE): média ponderada de todas as condições avaliadas.

As condições de teste descritas podem ser visualizadas na Figura 2.

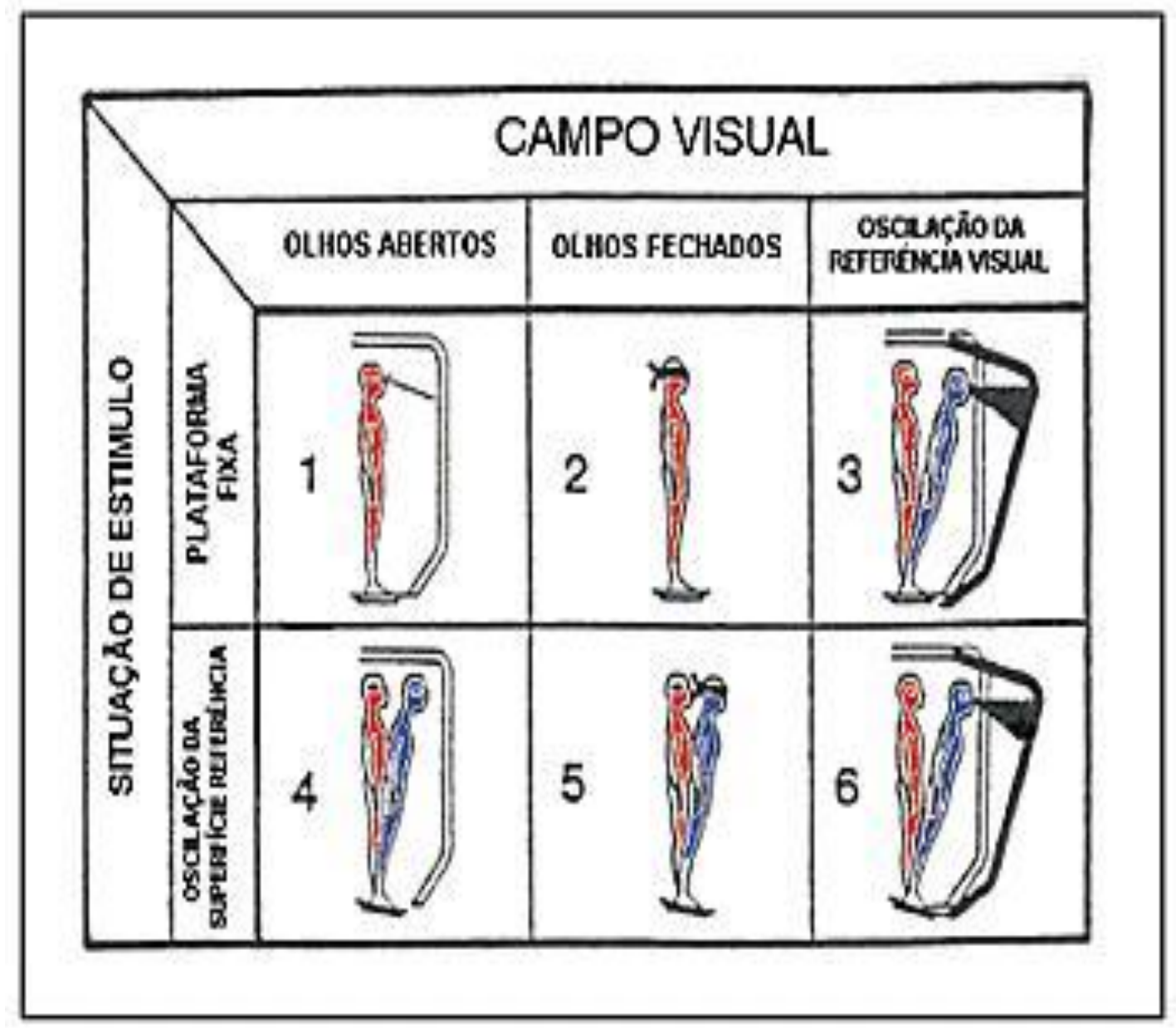

Figura 2 - Condições de estimulação sensorial no Teste de Integração Sensorial (TIS) Fonte: Bittar, 2007, p. 332. 
Os 24 pacientes foram avaliados de uma forma geral e depois divididos em dois grupos, com a finalidade de investigar a influência da presença de função vestibular pré-operatória na evolução do equilíbrio:

- pacientes com resposta negativa: arreflexos, com PC fria e quente sem resposta, e PRPD ausente em todas as frequências;

- pacientes com resposta positiva: resposta presente na PC e PRPD.

\subsection{Estatística}

O estudo estatístico consistiu em análise de variáveis quantitativas e qualitativas, e o programa utilizado para análise estatística foi o Statistical Package for the Social Sciences, SPSS versão 16.0.

Foram consideradas as variáveis de estudo: gênero, idade, lado implantado, função vestibular, presença ou ausência de resposta na PRPD, presença ou ausência de zumbido, presença ou ausência de sintomas otoneurológicos, avaliação das condições posturográficas. Todos os dados foram catalogados em fichas individuais contendo a identificação dos pacientes.

As variáveis foram analisadas levando-se em conta a verificação de diferenças entre os cinco momentos de avaliação. Os pontos de análise foram: o impacto do IC na organização sensorial do equilíbrio medida pela 
posturografia dinâmica e a diferença de resultados numéricos entre os cinco momentos da avaliação.

Para a avaliação da função vestibular, PRPD, sintomas otoneurológicos e do zumbido foi utilizado o teste de Wilcoxon. O teste exato de Fisher foi empregado para avaliar a influencia do lado implantado no zumbido, na PRPD e nos sintomas otoneurológicos. Para a avaliação das condições posturográficas foi utilizado o teste de Wilcoxon e Mann-Whitney. Esses testes foram utilizados porque as variâncias não eram homogêneas e a distribuição não era normal. Foi aplicado o teste de Kolmogorov-Smirnov e comprovou-se que não havia ajuste à distribuição normal.

Para as conclusões foi considerado um nível de significância de $5 \%$ $(p=0,05)$. 
5 RESULTADOS 


\section{RESULTADOS}

Foram avaliados 24 indivíduos que preencheram os critérios de inclusão durante o período de três anos. A amostra foi composta por 10 pacientes $(41,7 \%)$ do gênero feminino e 14 pacientes $(58,3 \%)$ do gênero

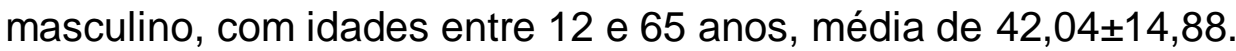

O grupo de estudo foi formado por 14 orelhas esquerdas $(58,3 \%)$ e 10 orelhas diretas $(41,7 \%)$ implantadas. As causas de surdez identificadas estão listadas na Tabela 1.

Tabela 1 - Causas da surdez dos sujeitos submetidos ao IC na amostra avaliada

\begin{tabular}{l|c|c}
\hline \hline Causa & $\begin{array}{c}\text { Quantidade de } \\
\text { pacientes }\end{array}$ & $\%$ \\
\hline Idiopática & 8 & 33,3 \\
Meningite & 4 & 16,7 \\
TCE & 2 & 8,2 \\
Otoesclerose & 2 & 8,2 \\
$\quad$ otoesclerose + Ménière & 1 & 4,2 \\
$\quad$ otoesclerose + AVC & 1 & 4,2 \\
Meningite + TCE & 1 & 4,2 \\
Sarampo + malária & 1 & 4,2 \\
História familiar de surdez + cirurgia de aneurisma & 1 & 4,2 \\
$\quad$ cerebral & & 4,2 \\
Síndrome de Guilian-Barré & 1 & 4,2 \\
Otite média crônica bilateral & 1 & 4,2 \\
Hemossiderose & 1 & 100 \\
\hline Total & 24 & \\
\hline \hline \% = porcento; AVC = acidente vascular cerebral; TCE = Traumatismo Crânio Encefálico &
\end{tabular}

\% = porcento; AVC = acidente vascular cerebral; TCE = Traumatismo Crânio Encefálico 


\subsection{Avaliação clínica}

\subsubsection{Zumbido}

A presença ou ausência de zumbido foi observada em todos os momentos de avaliação. Descrevemos a seguir a ocorrência do sintoma em cada uma delas.

\section{Dia 0}

Dentre os 24 indivíduos do grupo de estudo, três pacientes $(12,5 \%)$ negaram a presença de zumbido. Os demais 21 pacientes $(87,5 \%)$ relatavam a queixa.

\section{Dia 60}

Nove pacientes $(37,5 \%)$ não referiram zumbido enquanto $15(62,5 \%)$ deles tinham a queixa no primeiro retorno pós IC.

\section{Dia 120}

Doze pacientes (50\%) negaram zumbido no segundo retorno pósoperatório e 12 deles apresentavam o sintoma (50\%).

\section{Dias $180 / 365$}

Quatorze (58,3\%), entre os 24 pacientes, negavam a queixa e 10 $(41,7 \%)$ referiam zumbido nos terceiro e quarto retornos (Gráfico 1).

Em todos os indivíduos o zumbido foi caracterizado como bilateral e constante. Nenhum deles relatou piora do zumbido ao longo das avaliações. Em todos os momentos houve diferença estatística em relação à primeira avaliação pré IC $(p<0,05)$. 
Gráfico 1 - Ocorrência de zumbido em todos os momentos de avaliação e significância $(p)$ da diminuição do evento em relação à avaliação pré IC.

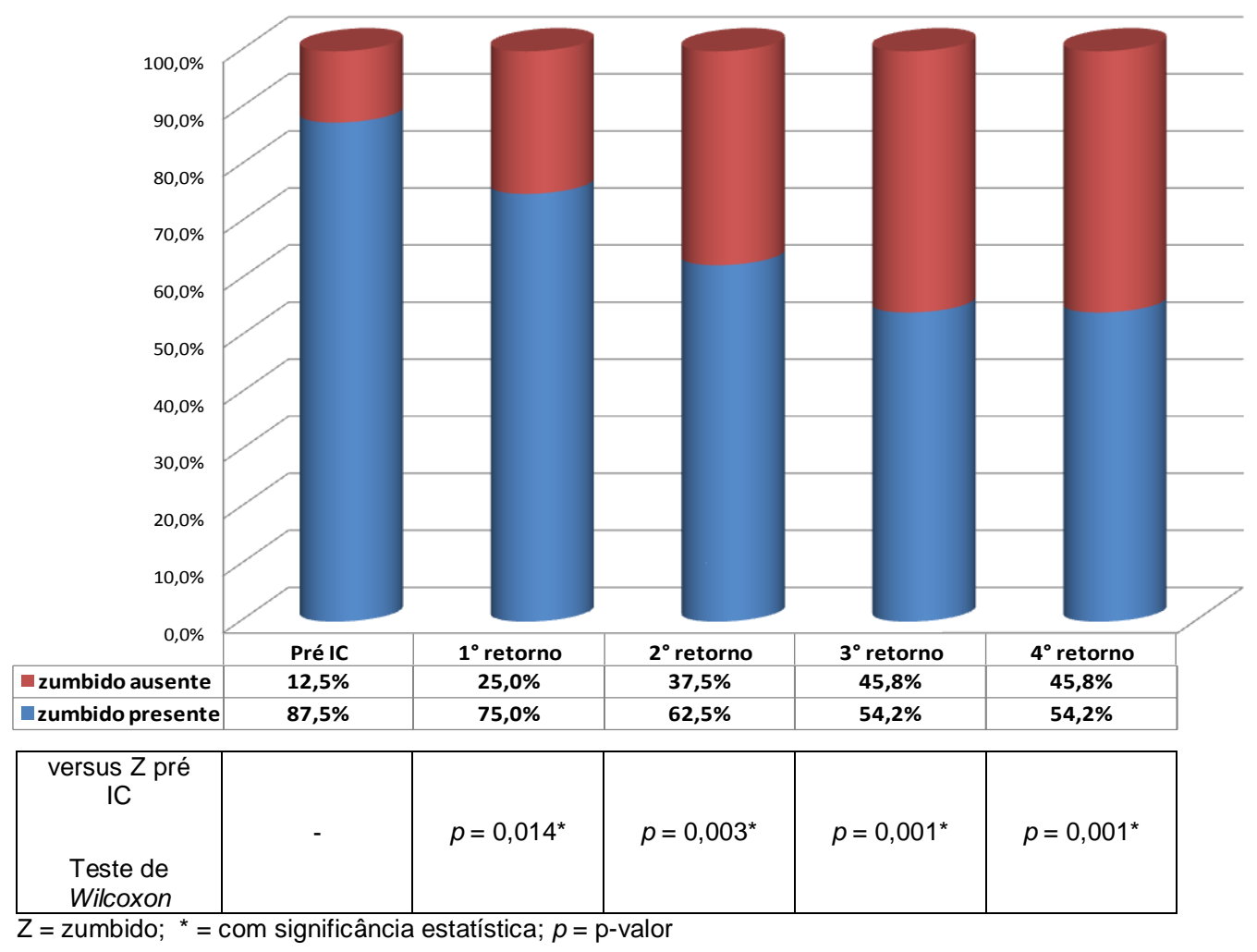

\subsubsection{Tontura}

Os resultados foram obtidos com base no questionário da anamnese (Anexo A) aplicados aos 24 pacientes ao longo de todas as avaliações.

\section{Dia 0}

Em 45,8\% dos casos pré IC (11 pacientes) não houve relato de qualquer queixa, já em $54,2 \%$ dos casos (13 pacientes) houve queixa de distúrbio do equilíbrio. 


\section{Dia 60}

Entre os 13 pacientes com a queixa pré IC houve melhora em cinco (38,5\%), piora em três (23\%) e em cinco o sintoma ficou inalterado (38,5\%).

\section{Dia 120}

No segundo retorno, tivemos oito casos de melhora $(61,5 \%)$ entre os 13 , um de piora $(7,7 \%)$ e quatro inalterados $(30,8 \%)$.

\section{Dia 180}

No terceiro retorno, 10 pacientes (76,9\%) melhoraram, dois pioraram $(15,4 \%)$ e um persistiu com a queixa $(7,7 \%)$.

\section{Dia 365}

Ao final do estudo, a tontura referida por 13 entre os 24 pacientes estudados pré IC melhorou em 11 (84,6\%); piorou em um $(7,7 \%)$ e em um permaneceu inalterada (7,7\%) (Gráfico 2).

Quando comparadas as avaliações do pré IC (Dia zero) com cada uma das avaliações pós-operatórias houve diferença estatisticamente significativa a partir do dia 120. Sete pacientes não apresentaram qualquer tipo de tontura ao longo das cinco avaliações realizadas e apenas dois relataram queixa em todas as avaliações. Os 15 pacientes restantes queixaram-se de tontura em um ou mais momentos das avaliações (Anexo B).

Dos 24 pacientes apenas cinco $(20,8 \%)$ apresentaram tontura no pósoperatório imediato e que melhorou logo no primeiro mês após a cirurgia. Dentre essas cinco pessoas, três já tinham queixa pré IC e duas não a apresentavam (Anexo B). 
A caracterização e as causas da tontura estão listadas no Anexo B.

Gráfico 2 - Ocorrência de tontura em todos os momentos de avaliação e significância $(p)$ da diminuição do evento em relação à avaliação pré IC.

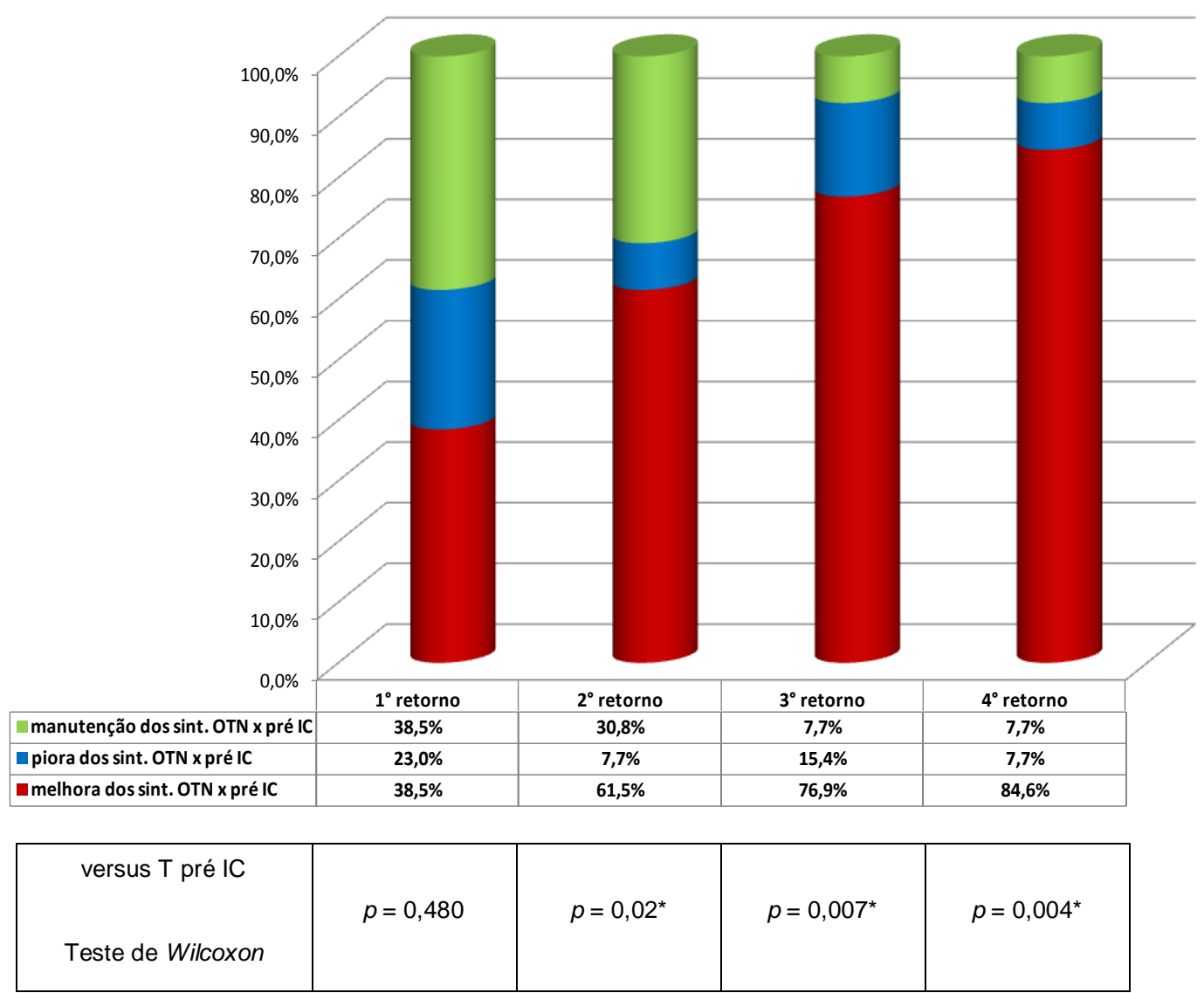

$\mathrm{T}=$ tontura; IC = Implante coclear; Sint. OTN = sintomas otoneurológicos; ${ }^{*}=$ valor apresenta significância estatística; $p=\mathrm{p}$-valor

\subsection{Exames computadorizados}

\subsubsection{Prova rotatória pendular decrescente}

Dentre os 24 indivíduos avaliados, 17 (70,8\%) apresentaram resposta e sete $(29,2 \%)$ não responderam ao primeiro teste ou aos subsequentes. Os indivíduos que apresentavam respostas mantiveram-nas e os que não as exibiam, não as adquiriram. 


\subsubsection{Eletronistagmografia}

Não foi observado comprometimento da oculomotricidade em qualquer momento das avaliações dos 24 pacientes estudados.

\section{Prova calórica}

Avaliando-se o nistagmo pós-calórico observamos seis casos de arreflexia pós-calórica bilateral (25\%), três hiporreflexia bilateral $(12,5 \%)$ e sete $(29,2 \%)$ normorreflexia bilateral. Assim se mantiveram durante as cinco avaliações. Oito pacientes $(33,3 \%)$ apresentaram diferentes funções em suas orelhas. Apenas dois desses oito indivíduos mantiveram a função inalterada ao longo de todas as avaliações, enquanto que seis deles apresentaram variabilidade da função vestibular no período de seguimento (Anexo C).

Com relação às orelhas implantadas, notamos que, comparando-se o Dia 0 com o Dia 365, houve piora em duas orelhas, quatro melhoraram e 18 não apresentaram mudança da função vestibular $(p=0,414)$. Entre as orelhas não implantadas, houve piora da função vestibular em quatro, melhora em três e as outras dezessete mantiveram a mesma resposta tanto no Dia 0 quanto no Dia 365 pós IC $(p=0,705)$. Os dados podem ser observados na Tabela 2. 
Tabela 2 - Avaliação da função vestibular em orelha implantada e orelha não implantada no Dia 365 ao comparar com o Dia 0

\begin{tabular}{l|c|c}
\hline \hline \multirow{2}{*}{ Função vestibular } & \multicolumn{2}{|c}{ Dia 365 } \\
\cline { 2 - 3 } & Orelha com IC & Orelha sem IC \\
\hline Piora & 2 & 4 \\
Melhora & 4 & 3 \\
Inalterada & 18 & 17 \\
TOTAL & 24 & 24 \\
\hline$p$ & 0,414 & 0,705 \\
\hline \hline
\end{tabular}

$\overline{\mathrm{IC}}=$ Implante coclear; $p=\mathrm{p}$-valor

5.2.3 Posturografia dinâmica computadorizada

Quando analisamos as condições posturográficas (C1 a C6), em relação à avaliação pré-operatória observamos que as Condições 3, 4, 5, 6 e IE apresentaram mudança ao longo das cinco avaliações (Tabela 3) em todos os pacientes. 
Tabela 3 - Valor de $p$ nas Condições 1 a 6 da PDC nos Dias 60, 120, 180 e 365 em relação ao Dia 0

\begin{tabular}{|c|c|c|c|c|}
\hline \multirow{2}{*}{$\begin{array}{l}\text { Condição e } \\
\text { significância }\end{array}$} & \multicolumn{4}{|c|}{ Dia } \\
\hline & 60 & 120 & 180 & 360 \\
\hline
\end{tabular}

$\begin{array}{lllll}\mathbf{C} 1 & & & & \\ \boldsymbol{p} & 0,837 & 0,648 & 0,824 & 0,898\end{array}$

\section{C2}

$\begin{array}{lllll}\boldsymbol{p} & 0,794 & 0,345 & 0,531 & 0,681\end{array}$

C3

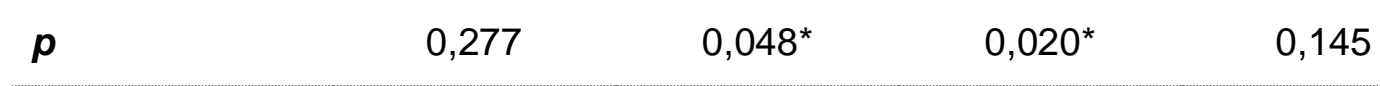

C4

$\begin{array}{lllll}p & 0,000^{*} & 0,000^{*} & 0,000^{*} & 0,000^{*}\end{array}$

C5

$\begin{array}{lllll}p & 0,433 & 0,013^{*} & 0,006^{*} & 0,004^{*}\end{array}$

C6

$\begin{array}{lllll}\boldsymbol{p} & 0,328 & 0,124 & 0,025^{*} & 0,015^{*}\end{array}$

IE

$\begin{array}{lllll}p & 0,026^{*} & 0,001^{*} & 0,000^{*} & 0,000^{*}\end{array}$

$\overline{\mathrm{PDC}}=$ Posturografia dinâmica computadorizada; $p=\mathrm{p}$-valor; ${ }^{*}=$ valor apresenta significância estatística

$\mathrm{Na}$ Condição 3 da PDC encontramos valor estatisticamente significativo no Dia $120(p=0,048)$ e $180(p=0,020)$. Já para a Condição 4 houve diferença em todos os momentos de avaliação em relação ao Dia 0 com $p=0,000$ nos Dias 60, 120, 180 e 365. Quando considerada a Condição 
5 da PDC (C5) observamos aumento numérico estatisticamente significativo entre o Dia 0 e os Dias $120(p=0,013), 180(p=0,006)$ e $365(p=0,004)$. Em relação à Condição 6 verificamos aumento numérico significativo apenas no Dia $180(p=0,025)$ e Dia $365(p=0,015)$. O mesmo aconteceu em relação ao índice final de equilíbrio (IE), que apresentou melhora significativa em relação ao Dia 0; no Dia 60 ( $p=0,026)$; no Dia 120 ( $p=0,001)$; nos Dias 180 e $365(p=0,000)$. (Demais informações no Anexo D)

5.2.4 Posturografia dinâmica computadorizada vs. prova calórica

Quando comparamos os resultados obtidos na PDC e a resposta póscalórica pudemos documentar que houve diferença significante na evolução das condições posturográficas entre os pacientes arreflexos, ou seja, os pacientes que não apresentaram resposta à $\mathrm{PC}$ e com PRPD ausente (resposta negativa) e aqueles que apresentavam resposta nessas provas (resposta positiva) ao início da avaliação sequencial (Tabela 4).

5.2.5 Comparação entre os grupos com resposta positiva e negativa

Avaliando as condições de plataforma fixa C1, C2 e C3, observamos que no Dia 0, anterior ao IC, os dois grupos: resposta positiva e resposta negativa eram estatisticamente diferentes apenas em relação a C1 $(p=0,048)$. No Dia 365 , após um ano da cirurgia mostraram-se semelhantes em todas as condições $\mathrm{C} 1, \mathrm{C} 2, \mathrm{C} 3$. Com relação às condições de 
plataforma móvel, dependentes da informação vestibular, os grupos se diferenciaram em diversos momentos. Os grupos foram semelhantes nas duas medidas de $\mathrm{C} 4$, mas se diferenciaram estatisticamente no Dia 0 em C5 $(p=0,008)$ e C6 $(p=0,008)$. Mantiveram a diferença no Dia 365 nas duas condições C5 $(p=0,007)$ e C6 $(p=0,029)$. Essa mesma diferença foi observada em IE no Dia $0(p=0,004)$ e Dia $365(p=0,024)$. (Demais informações no Anexo E)

\subsubsection{Evolução dos grupos com resposta positiva e negativa}

Analisando os grupos nas condições de plataforma fixa, observamos que apenas o grupo com resposta negativa apresentou diferença significante entre o Dia 0 e o Dia 365 em C3 $(p=0,028)$. As demais condições mantiveram-se inalteradas.

Com relação às condições de plataforma móvel, observamos várias diferenças entre o grupo com resposta positiva e o de resposta negativa. 0 grupo com resposta positiva apresentou diferença significativa entre o Dia 0 e o Dia 365 em C4 $(p=0,001), C 5(p=0,008)$ e C6 $(p=0,033)$. No entanto, o grupo com resposta negativa só apresentou diferença significativa em $\mathrm{C} 4$ $(0,018)$ para a mesma avaliação. Essas diferenças repercutiram no IE de ambos os grupos com $p=0,005$ para o grupo de resposta positiva e $p=0,018$ para o grupo de resposta negativa. 
Tabela 4 - Valor de $p$ na comparação entre pacientes portadores de arreflexia pós-calórica e pacientes com resposta na PC nos Dias 0 e 365

\begin{tabular}{|c|c|c|c|c|}
\hline & PC & Resposta negativa & Resposta positiva & $p$ \\
\hline & Dia 0 & & & $0,048^{*}$ \\
\hline \multirow[t]{3}{*}{ Condição 1} & Dia 365 & & & 0,292 \\
\hline & $p$ & 0,176 & 0,356 & - \\
\hline & Dia 0 & & & 0,172 \\
\hline \multirow[t]{3}{*}{ Condição 2} & Dia 365 & & & 0,324 \\
\hline & $p$ & 0,080 & 0,477 & - \\
\hline & Dia 0 & & & 0,324 \\
\hline \multirow[t]{3}{*}{ Condição 3} & Dia 365 & & & 0,848 \\
\hline & $p$ & $0,028^{*}$ & 0,794 & - \\
\hline & Dia 0 & & & 0,061 \\
\hline \multirow[t]{3}{*}{ Condição 4} & Dia 365 & & & 0,086 \\
\hline & $p$ & $0,018^{*}$ & $0,001^{*}$ & - \\
\hline & Dia 0 & & & $0,008^{*}$ \\
\hline \multirow[t]{3}{*}{ Condição 5} & Dia 365 & & & $0,007^{*}$ \\
\hline & $p$ & 0,18 & $0,008^{*}$ & - \\
\hline & Dia 0 & & & $0,008^{*}$ \\
\hline \multirow[t]{3}{*}{ Condição 6} & Dia 365 & & & $0,029^{*}$ \\
\hline & $p$ & 0,180 & $0,033^{*}$ & - \\
\hline & Dia 0 & & & $0,004^{*}$ \\
\hline \multirow[t]{2}{*}{ IE } & Dia 365 & & & $0,024^{*}$ \\
\hline & $p$ & $0,018^{*}$ & $0,005^{*}$ & - \\
\hline
\end{tabular}

$\overline{\mathrm{PC}}=$ Prova calórica; ${ }^{*}=$ valor apresenta significância estatística; IE = Indice final de equilíbrio 
6 DISCUSSÃO 


\section{DISCUSSÃO}

Para avaliarmos a interferência do IC sobre a função vestibular, de acordo com o nosso projeto foram pesquisados sequencialmente pacientes adultos, de ambos os gêneros com surdez pós-lingual. Optamos por avaliar desta maneira para facilitar a coleta de dados e a realização dos exames. Levamos, também, em conta o fato de que esses indivíduos apresentaram memória auditiva, fato marcante no reconhecimento de sons externos.

As causas da surdez em nossa amostra de sujeitos foram diversas, com destaque para os casos de surdez idiopática, meningite, TCE e otoesclerose, como constatado por outros autores nas amostras que analisaram (Eisenberg et al., 1982; Huygen et al., 1995; Buchman et al., 2004; Suarez et al., 2007; Hansen et al., 2010). A etiologia da surdez é importante para a avaliação da função vestibular residual. Em nossa amostra, a meningite e o TCE estavam relacionados a cinco $(71,4 \%)$ dos sete casos que apresentaram arreflexia vestibular bilateral. Nossos achados estão de acordo com Vibert et al. (2001), que relacionam a arreflexia vestibular à meningite, enquanto em outras etiologias é comum observar-se a presença de função vestibular residual.

Para uma boa avaliação torna-se importante detalhar as queixas otoneurológicas (Krause et al., 2009c). No entanto, optamos por não utilizar os questionários validados tradicionais como o $\mathrm{DHI}$ por sua complexidade e extensão. Lançamos mão do questionário utilizado no Serviço de 
Otoneurologia do HC-FMUSP com a finalidade de identificar quais pacientes tinham tontura ou não nas cinco avaliações.

É nítida a influência do IC na evolução do zumbido. Por esse motivo incluímos esse dado, embora não seja o foco de nossa pesquisa. Nossos achados demonstram redução significativa da queixa ao longo do período de estudo, fato confirmado por diversos autores na literatura (Ito, Sakakihara, 1994; Ito, 1997; Fukuda et al., 2000; Nita et al., 2002; Kim et al., 2013). Nenhum paciente relatou piora do zumbido ao longo das avaliações. Após um ano de avaliação, o sintoma estava presente em 41,7\% da amostra (10 sujeitos) e naqueles que ainda o percebiam, o fato ocorreu apenas com o IC desligado. A nova aferência auditiva oferecida pelo IC foi decisiva na melhora e resolução do sintoma. De fato, o efeito benéfico do IC no zumbido poderia ser explicado pelo mascaramento produzido pelas sensações auditivas ou pela própria estimulação elétrica das vias auditivas levando a uma reorganização das vias centrais (Nita et al., 2002; Palau et al., 2010).

A tontura estava presente na avaliação pré IC em 13 indivíduos, cifra correspondente a $54,2 \%$ da amostra. Os indivíduos com arreflexia vestibular referiam dificuldade para deambular em ambientes escuros ou com pouca luz. No caso de ocorrência da tontura no pós-operatório imediato observouse que dos 24 pacientes avaliados, apenas cinco (20,8\%) apresentaram tontura no pós-operatório imediato, três pacientes tinham a queixa no pré IC e dois não a tinham. E essa queixa melhorou até um mês após a cirurgia, em concordância com Rossi et al. (1998), Kubo et al. (2000), Bouccara et al. (2005) e Krause et al. (2009a). A melhora clínica de uma lesão vestibular 
aguda em indivíduo saudável é esperada após um mês da lesão, segundo as teorias de compensação vestibular (McCabe et al., 1972; Lacour et al., 2009).

Um ano após o IC observamos melhora significativa do sintoma em 11 pacientes $(45,8 \%$ da amostra) em comparação ao Dia $0 \quad(p=0,004)$. Podemos atribuir essa melhora ao processo de compensação vestibular, opinião também compartilhada por Buchman et al. (2004), Filipo et al. (2006), Bonucci et al. (2008) e Krause et al. (2009a). Os dados suportam a indicação de um seguimento da função vestibular em longo prazo, uma vez que estudos com período de seguimento curto ou não definido, não são capazes de determinar a real influência da estimulação do IC no equilíbrio corporal como refere Abramides et al. (2009).

Acreditamos que a compensação vestibular que ocorre nesses pacientes seja secundária a um mecanismo dinâmico e complexo que compreende a participação de vários processos e informações: informação visual, função labiríntica, aferência e resposta do sistema somatosensorial, sinapses entre fibras, substituição de neurotransmissores, etc. O surgimento de uma nova aferência auditiva provoca mudanças na circuitaria neural do equilíbrio, e sua consequente reorganização. Essa mudança estrutural das vias pôde ser documentada em nossos pacientes a partir de seis meses da colocação do IC.

Notamos que alguns dos pacientes que evoluíram com tontura pós IC apresentavam comorbidades que podem ter sido a causa da 
descompensação vivida naquele momento, que não propriamente o trauma cirúrgico (Bittar et al., 2007). Identificamos sujeitos portadores de enxaqueca, distúrbio do metabolismo de carboidratos, síndrome cervical, tontura crônica subjetiva, VPPB - causas reconhecidamente capazes de interferir na recuperação vestibular (Bittar et al., 2007). É possível que tenha ocorrido ainda a reativação de diagnósticos prévios, como a doença de Ménière. Portanto, é fundamental a avaliação otoneurológica no pré IC, uma vez que existem condições associadas que podem comprometer o equilíbrio do paciente pós IC. Segundo Steenerson et al. (2001) a avaliação e acompanhamento da função vestibular pré e pós IC deve ser realizada de rotina.

Não há consenso na literatura quanto à melhor forma de avaliação da função vestibular nos sujeitos submetidos ao IC. No entanto, a oculografia é indicação unânime na pesquisa e avaliação do reflexo vestíbulo-ocular. Por meio da pesquisa do nistagmo pós-calórico identificamos as diversas características funcionais da resposta vestibular: arreflexia, hiporreflexia, normorreflexia ou hiperreflexia. Buchman et al. (2004), Jacot et al. (2009), entre muitos outros autores, utilizaram provas rotatórias por apresentarem a possibilidade de avaliação do RVO em diversas frequências e velocidades de estimulação. A avaliação rotatória torna-se obrigatória naqueles indivíduos que apresentaram arreflexia, pois a resposta pós-calórica encontra-se em níveis baixos de frequência, que não são correspondentes às solicitações de movimentos necessárias às tarefas da vida diária. 
É importante comentar que, nem sempre uma função vestibular pobre, documentada por exames, foi acompanhada pela queixa de tontura provável resultado da compensação central do equilíbrio decorrentes do processo de habituação e neuroplasticidade. Apesar da difícil avaliação, notamos que houve ligeira queda da VACL no Dia 60 em relação à avaliação pré IC, com recuperação após o Dia 180. Essas observações confirmam o sofrimento vestibular imediato após IC de acordo com Mangham (1987), mas demonstram, também, a recuperação funcional alcançada após seis meses, atribuída à compensação central.

A importância da documentação da função vestibular antes da cirurgia de IC reside em três aspectos principais: conhecimento de um possível labirinto arreflexo, que norteia o lado a ser implantado (Huygen et al., 1995); a condução de possíveis sintomas vestibulares durante o período pósoperatório (Filipo et al., 2006); e, por último, o prognóstico desse sujeito no que se refere ao equilíbrio corporal, como veremos a seguir.

Ao avaliar os casos individualmente, entre os seis pacientes que sofreram mudança da função vestibular entre a avaliação pré IC e após um ano, observamos alguns fatos interessantes e formulamos algumas hipóteses para explicá-los.

1. MBS apresentava ausência de resposta pós-calórica pós-meningite e evoluiu para hiporreflexia bilateral ao final de um ano. É possível que houvesse função vestibular residual mínima, não detectada pelos métodos de avaliação. Mas a hipótese que melhor condiz com a 
situação apresentada é considerar que o estímulo gerado pelo IC foi capaz de reestruturar a via vestibular por meio da aferência auditiva. As conexões presentes na via foram estimuladas e contribuíram para o melhor controle postural. A melhora observada na orelha contralateral pode ser facilmente explicada pela estimulação obtida por fibras intercomissurais, que permitem o compartilhamento de informações, ação fundamental na compensação vestibular (Baloh, Honrubia, 2001).

2. A mesma hipótese pode ser considerada nos casos de SBS, ESC, DBS e CRSC que também apresentaram melhora da função vestibular após um ano.

3. No caso de ACS, após queixa de desequilíbrio durante a primeira semana depois do IC, foi observada a perda da resposta pós calórica na orelha implantada, situação anteriormente relatada em literatura (Enticott et al.,2006; Filipo et al., 2006). Neste caso, a orelha não implantada, anteriormente hiporreflexa, evoluiu para arreflexia. Fato interessante é que, em nenhum dos momentos da avaliação, quando foi feita a medida da função vestibular, o paciente tenha referido desequilíbrio e/ou tontura. Nesse caso, podemos considerar a evolução clínica à luz da compensação central. Segundo Lacour et al. (2009) a plasticidade neuronal envolvida no processo de compensação vestibular depende não apenas do tipo de perda (funcional ou estrutural), mas também da forma como essa lesão foi instalada. As perdas graduais ou transitórias de função são 
compensadas de forma mais efetiva do que perdas agudas e súbitas. Isto ocorre porque há toda uma preparação do sistema neural, gerando sinapses, modificando neurotransmissores, fabricando proteínas, etc., com a finalidade de restaurar a função primordial de manter o equilíbrio. Todo esse processo necessita de tempo, não disponível nas lesões agudas.

Nenhum outro exame utilizado para avaliação quantitativa do equilíbrio corporal é tão útil como a posturografia dinâmica computadorizada. A PDC demonstrou de forma clara e consistente a evolução gradual do equilíbrio corporal de cada paciente ao longo de todas as avaliações. Assim como nós, Buchman et al. (2004) também constataram melhor desempenho postural pós IC por meio de PDC em longo prazo. Em todos os momentos do acompanhamento, pudemos constatar a melhora global do equilíbrio corporal, medida pelo IE. Essa evolução positiva do equilíbrio global, no entanto, não ocorreu de forma homogênea em todas as condições da PDC.

O primeiro sinal de melhora no desempenho postural, foi observado na Condição 4, solo instável com fixação visual, que evoluiu de maneira significativa em todos os momentos a partir da primeira avaliação, sugerindo que há melhor aproveitamento visual já a partir do segundo mês após a cirurgia de IC. Em 120 dias constatamos maior efetividade do desempenho vestibular na Condição 5 e pudemos notar que a Condição 3, solo fixo com conflito visual, também mostrou melhora significante sugerindo que a estabilidade postural evoluiu positivamente, mesmo em situações de conflito. Schwab e col. (2010) também observaram melhora da função vestibular a 
partir das Condições 4 e 5 . A situação máxima de conflito de informações ambientais é simulada pela Condição 6, com solo e visor instáveis. Essa condição só apresentou melhora efetiva a partir do sexto mês e assim se manteve até o final da observação. As nossas observações da evolução posturográfica dos pacientes, indica uma hierarquia de aprendizado e aproveitamento da nova informação sensorial, que se completa aos 180 dias. Primeiro na percepção de uma melhor informação vestibular, depois na sua integração central às aferências sensoriais e, finalmente, no aproveitamento global de todas as informações recebidas, novas e antigas. Essa hierarquia observada reproduz a evolução natural da compensação central, conforme já descrito por vários autores. Primeiro as limitações estáticas são resolvidas pelo balanceamento da atividade elétrica no núcleo vestibular. Posteriormente, ocorre a restauração dos processos dinâmicos, que dependem da integração e substituição de informações provenientes das várias redes neurais participantes do sistema de manutenção do equilíbrio corporal que chamamos de cognição. (McCabe et al., 1972; Newlands et al., 2005; Lacour et al., 2006).

Constatamos, portanto, que há efetiva melhora do equilíbrio corporal documentada pela PDC ao longo de um ano, nos indivíduos que receberam IC. Considerável parte dessa melhora foi atribuída ao melhor desempenho na Condição 5, estratégia tipicamente vestibular, no grupo avaliado. No entanto, resta-nos saber se há diferença no desempenho vestibular ao longo do tempo entre aqueles que possuem e os que não possuem função vestibular pré IC. Para tanto dividimos os pacientes em dois grupos segundo 
a resposta à prova calórica: sem função vestibular (arreflexia vestibular) e aqueles com função vestibular residual presente. Quando esses grupos foram comparados entre si, observamos que diferiam no tipo de estratégias posturais utilizadas ao longo do estudo.

Os grupos se diferenciaram significativamente na avaliação pré IC nas Condições 5 e 6, e mantiveram essa diferença após um ano. O fato é facilmente explicável, pois o grupo com arreflexia vestibular não possuía aferência vestibular - condição necessária ao bom desempenho nos desafios posturais apresentados por essas condições.

Durante o acompanhamento, apenas o grupo com arreflexia vestibular apresentou diferença significativa nas condições de plataforma fixa entre a avaliação pré IC e a última avaliação depois de um ano. Os achados sugerem que houve melhora da estabilidade postural, mesmo em condições de conflito visual como consequência à aquisição da nova informação fornecida pelo IC. O mesmo não aconteceu com aqueles que possuíam resíduos vestibulares, que já apresentavam bom desempenho nessas mesmas condições e não se modificaram.

Com relação às condições de plataforma móvel, aqueles que possuíam resíduos vestibulares apresentaram diferença significativa entre a avaliação pré IC e um ano após. As condições de plataforma móvel provocam respostas somatossensoriais conflitantes e levam à queda na ausência de informação vestibular adequada. Os indivíduos foram capazes de aproveitar a nova aferência auditiva e melhorar o desempenho vestibular. 
No entanto, no grupo com arreflexia vestibular, a única melhora observada ao final do experimento foi na Condição 4, sugerindo melhor aproveitamento da informação visual. Os sujeitos não puderam melhorar o desempenho vestibular, uma vez que ele já não existia. O fator principal que contribuiu positivamente na recuperação postural foi o melhor aproveitamento da informação visual, sugerindo mais uma vez, a integração sensorial promovida pelo cérebro durante o período de compensação central. Segundo Lacour (2006) e Curthoys (2000) a falta de informações vestibulares culmina no aumento dos inputs visuais e somatosensoriais em um processo de substituição sensorial.

Por fim, ambos os grupos apresentaram diferença significativa no IE, retratando o melhor desempenho das estratégias de recuperação postural. Entretanto, notamos que a melhora do índice foi expressivamente maior no grupo que possuía função vestibular $(p=0,005)$ em relação ao grupo com arreflexia vestibular $(p=0,018)$. Situação facilmente entendida, uma vez que os indivíduos com arreflexia vestibular só puderam contar com a integração sensorial durante o período de avaliação e melhorar a Condição 4, enquanto que os sujeitos que apresentavam resposta à prova calórica pré IC foram capazes de melhorar todas as condições de plataforma móvel. 
7 CONCLUSÕES 


\section{CONCLUSÕES}

Conclui-se que:

Questão 1: Presença e evolução da tontura e do zumbido antes e após o IC

- $20,8 \%$ dos casos de nossa amostra apresentou tontura no pósoperatório imediato que foi resolvida em 30 dias;

- Nos casos em que havia tontura pré-existente ao IC houve melhora em $84,6 \%$, piora em $7,7 \%$ e não houve alteração em $7,7 \%$ ao final de um ano.

- Não houve relato de piora do zumbido ao longo das avaliações. Ao final do estudo $41,7 \%$ referia essa queixa, enquanto $58,3 \%$ não relatava a presença de zumbido.

Questão 2: Influência da presença de função vestibular pré-operatória na evolução do equilíbrio

- A ausência de resposta pós-calórica na avaliação pré-operatória implicou em pior prognóstico na evolução do equilíbrio corporal ao longo de um ano.

Questão 3: Melhora da postura por meio da posturografia dinâmica computadorizada (PDC)

- A evolução do equilíbrio manteve-se no tempo habitualmente esperado para 0 desenvolvimento dos mecanismos de compensação central; 
- O melhor desempenho obtido em todas as condições posturográficas foi atingido em seis meses e mantido até a avaliação final de um ano. 
ANEXOS 


\section{ANEXO A}

\section{ANAMNESE}

\section{ETIQUETA DE IDENTIFICAÇÃO}

\section{Tontura}

Início:

Tipo - ( ) Rotatória ( ) Flutuação ( ) Lateropulsão

$\begin{array}{lllll}\text { Frequência - ( ) Crise } & \text { ( ) Persistente } & & \\ \text { Duração - } & \text { ( ) Segundos } & \text { ( ) Minutos } & \text { ( ) Horas } & \text { ( ) Dias } \\ \text { Intensidade - ( ) Leve } & \text { ( ) Moderada } & \text { ( ) Intensa } & \text { ( ) Variável } \\ \text { Ocorrência - ( ) Diária } & \text { ( ) Semanal } & \text { ( ) Mensal } & \text { ( ) Esporádica }\end{array}$

Relação com:

( ) movimento

( ) caminhada

( ) veiculo em movimento

( ) escurecimento visual

( ) perda de consciência

( ) exercícios físicos

( ) jejum

( ) cefaleia

( ) paroxismos

\section{Outros sintomas}

( ) náusea

( ) vômito

( ) quedas - ( ) D $\quad$ ( ) E $\quad$ ( ) F $\quad$ ( ) T 
( ) desvio da cabeça - ( ) D $\quad$ ( ) E $\quad$ ( ) F $\quad$ ( ) T

( ) desvio da marcha - ( ) D ( ) E

( ) zumbido - ( ) Lado ( ) Contínua ( ) Intermitente

( ) hipoacusia - ( ) Unilateral ( ) Bilateral Causa:

( ) plenitude auricular - ( ) Unilateral ( ) Bilateral

( ) hipersensibilidade auditiva

( ) dor cervical

( ) alteração do sono

( ) alteração da concentração

( ) alteração de memória

( ) alteração de humor

( ) interferência na vida social

\section{Antecedentes pessoais}

Doenças de base -

Uso de drogas -

Trauma -

Meningite -

Otites -

Exposição a ruído -

Internações -

Cirurgia -

Uso de AASI -

\section{Antecedentes familiares}

\section{Hábitos}

\section{Exame físico}

( ) Rinoscopia -

( ) Oroscopia -

( ) Otoscopia - 


\section{Avaliação neurológica}

\section{Resultados de exames}

( ) Exame audiométrico -

( ) Exames de imagem -

( ) Exames laboratoriais -

( ) Exame otoneurológico -

( ) Pesquisa do nistagmo espontâneo -

( ) Pesquisa do nistagmo semi-espontâneo -

( ) Pesquisa do nistagmo posicional -

( ) DLE ( ) TCE ( ) DLD ( ) TCD

( ) ROSE ( ) SENTADO

Rastreio - Tipo:

Optocinético -

$\begin{array}{rrr}\text { Prova calórica - } 30 \text { Graus } & \text { ( ) OD } & \text { ( ) OE } \\ -44 \text { Graus } & \text { ( ) OD } & \text { ( ) OE }\end{array}$

PDC -

PRPD -

Outros -

D - direito; E - esquerdo; F - frontal; T - traseiro; AASI - Aparelho de Amplificação Sonora Individual; DLE - Decúbito Lateral Esquerdo; TCE - Torção cervical esquerda: DLD Decúbito Lateral Direito: TCD - Torção cervical direita; ROSE - Posição de Rose: cabeça em hiperextensão; OD - orelha direita; OE - orelha esquerda; PDC - Posturografia Dinâmica Computadorizada; PRPD - Prova Rotatória Pendular Decrescente 
ANEXO B

CAUSAS, CARACTERÍSTICAS E OCORRÊNCIA DE TONTURA

EM TODOS OS MOMENTOS DE AVALIAÇÃO

\begin{tabular}{|c|c|c|c|c|c|c|c|c|c|}
\hline nome & idade & causa & tipo & $\begin{array}{l}\text { pós-op } \\
\text { imediato }\end{array}$ & dia zero & dia 60 & dia 120 & dia 180 & dia 365 \\
\hline M.B.S & 12 anos & $\begin{array}{l}\text { meningite } \\
\text { enxaqueca }\end{array}$ & $\begin{array}{l}\text { desvio de marcha, queda no } \\
\text { Romberg crises de vertigem } \\
\text { associada a enxaqueca }\end{array}$ & - & + & - & + & - & - \\
\hline C.A.S.P & 15 anos & meningite & $\begin{array}{l}\text { desvio de marcha, queda e } \\
\text { desequilíbrio pós meningite }\end{array}$ & + & + & + & - & - & - \\
\hline N.M.O & 23 anos & ausente & sem queixas & - & - & - & - & - & - \\
\hline A.L & 26 anos & meningite & $\begin{array}{l}\text { desequilibrio logo após } \\
\text { meningite apenas }\end{array}$ & - & + & + & - & - & - \\
\hline E.R.A.P & 27 anos & ausente & sem queixas & - & _ & - & - & - & - \\
\hline A.R.N & 28 anos & $\begin{array}{l}\text { Ménière } \\
\text { cinetose } \\
\text { enxaqueca }\end{array}$ & $\begin{array}{l}\text { dificuldade em deambular em } \\
\text { ambientes escuros ou com } \\
\text { pouca luz }\end{array}$ & - & + & - & - & - & - \\
\hline W.L.O & 29 anos & $\begin{array}{l}\text { meningite } \\
\text { TCE }\end{array}$ & $\begin{array}{l}\text { dificuldade em deambular em } \\
\text { ambientes escuros ou com } \\
\text { pouca luz }\end{array}$ & - & + & - & - & - & - \\
\hline A.A.C & 34 anos & ausente & sem queixas & - & - & _ & - & - & _ \\
\hline D.B.s & 37 anos & enxaqueca & $\begin{array}{l}\text { crises de vertigem associada a } \\
\text { enxaqueca }\end{array}$ & - & + & + & + & + & + \\
\hline C.R.S.C & 39 anos & ausente & sem queixas & - & _ & _ & _ & _ & _ \\
\hline M.O.P & 43 anos & $\begin{array}{l}\text { alteração de } \\
\text { metabolismo de } \\
\text { carbohidratos } \\
\text { enxaqueca } \\
\text { VPPB }\end{array}$ & $\begin{array}{l}\text { flutuação, crises de vertigem } \\
\text { associada a enxaqueeca e } \\
\text { tontura em decúbito lateral } \\
\text { direito }\end{array}$ & + & + & + & - & - & - \\
\hline J.P & 44 anos & $\begin{array}{l}\text { mudança do mapa } \\
\text { cortical devido } \\
\text { aferência auditiva }\end{array}$ & $\begin{array}{l}\text { desequilibrio ao andar de } \\
\text { bicicleta }\end{array}$ & - & - & - & + & - & - \\
\hline F.L.G & 44 anos & diabetes & flutuação & + & - & + & - & + & + \\
\hline A.C.S & 45 anos & ausente & sem queixas & - & - & - & - & - & - \\
\hline E.P.S & 46 anos & TCE & $\begin{array}{l}\text { desequilibrio e dificuldade em } \\
\text { deambular em ambientes } \\
\text { escuros ou com pouca luz }\end{array}$ & - & + & + & - & - & - \\
\hline S.B.S & 47 anos & $\begin{array}{l}\text { otoesclerose } \\
\text { Ménière }\end{array}$ & $\begin{array}{l}\text { crises de vertigem cessaram } \\
\text { com a surdez profunda }\end{array}$ & - & - & + & - & - & - \\
\hline L.F.L & 49 anos & $\begin{array}{l}\text { enxaqueca } \\
\text { síndrome cervical } \\
\text { alteração } \\
\text { Metabolismo de } \\
\text { carbohidratos }\end{array}$ & $\begin{array}{l}\text { desequilibrio, flutuação, crises } \\
\text { de vertigem associada a } \\
\text { enxaqueca }\end{array}$ & + & + & + & + & + & - \\
\hline S.N.P & 53 anos & ausente & sem queixas & - & _ & _ & - & - & _ \\
\hline J.N & 59 anos & TCS pós meningite & $\begin{array}{l}\text { desvio de marcha, tontura e } \\
\text { instabilidade }\end{array}$ & - & + & + & + & - & - \\
\hline G.M.M & 60 anos & ausente & sem queixas & - & + & - & - & - & - \\
\hline U.M.S & 61 anos & ausente & sem queixas & - & - & - & - & - & - \\
\hline E.S.C & 61 anos & pico hipertensivo & tontura & _ & + & - & - & - & - \\
\hline J.A.M & 62 anos & $\begin{array}{l}\text { AlT por pico } \\
\text { hipertensivo }\end{array}$ & tontura, desequilíbrio & + & - & + & - & - & - \\
\hline M.A.O.B & 65 anos & $\begin{array}{l}\text { pós cirurgia de } \\
\text { aneurisma }\end{array}$ & desequilíbrio & - & + & + & + & + & + \\
\hline
\end{tabular}

+: presente, -: ausente, TCE: traumatismo crânioncefálico, VPPB: vertigem postural paroxística benigna, TCS: tontura crônica subjetiva, AIT: ataque isquêmico transitório 
ANEXO C

RESPOSTAS DAS PROVAS CALÓRICAS NAS ORELHAS IMPLANTADAS E NÃO IMPLANTADAS NO DIA 0 E NO DIA 365

EM DESTAQUE, OS SEIS PACIENTES QUE TIVERAM MUDANÇA DO EXAME

\begin{tabular}{|c|c|c|c|c|c|c|}
\hline \multirow[b]{2}{*}{ Nome } & \multirow[b]{2}{*}{ Gênero } & \multirow{2}{*}{$\begin{array}{l}\text { Idade } \\
\text { (anos) }\end{array}$} & \multicolumn{2}{|c|}{ Dia 0} & \multicolumn{2}{|c|}{ Dia 365} \\
\hline & & & $\begin{array}{c}\text { Orelha } \\
\text { implantada }\end{array}$ & $\begin{array}{l}\text { Orelha não } \\
\text { implantada }\end{array}$ & $\begin{array}{c}\text { Orelha } \\
\text { implantada }\end{array}$ & $\begin{array}{l}\text { Orelha não } \\
\text { implantada }\end{array}$ \\
\hline M.B.S & M & 12 & 0 & 0 & $\downarrow$ & $\downarrow$ \\
\hline C.A.S.P & $\mathrm{F}$ & 15 & 0 & 0 & 0 & 0 \\
\hline N.M.O & $\mathrm{F}$ & 23 & $\mathrm{nl}$ & $\mathrm{nl}$ & $\mathrm{nl}$ & $\mathrm{nl}$ \\
\hline A.L & M & 26 & 0 & 0 & 0 & 0 \\
\hline E.R.A.P & M & 27 & $\mathrm{nl}$ & $\mathrm{nl}$ & $\mathrm{nl}$ & $\mathrm{nl}$ \\
\hline A.R.N & M & 28 & 0 & 0 & 0 & 0 \\
\hline W.L.O & M & 29 & 0 & 0 & 0 & 0 \\
\hline A.A.C & $\mathrm{F}$ & 34 & $\mathrm{nl}$ & $\mathrm{nl}$ & $\mathrm{nl}$ & $\mathrm{nl}$ \\
\hline D.B.S & $\mathbf{F}$ & 37 & nl & $\uparrow$ & nl & $\mathrm{nl}$ \\
\hline C.R.S.C & $\mathbf{F}$ & 39 & $\uparrow$ & $\uparrow$ & nl & $\mathrm{nl}$ \\
\hline M.O.P & $\mathrm{F}$ & 43 & $\mathrm{nl}$ & $\mathrm{nl}$ & $\mathrm{nl}$ & $\mathrm{nl}$ \\
\hline J.P & M & 44 & $\downarrow$ & $\downarrow$ & $\downarrow$ & $\downarrow$ \\
\hline F.L.G & M & 44 & $\mathrm{nl}$ & $\mathrm{nl}$ & $\mathrm{nl}$ & $\mathrm{nl}$ \\
\hline A.C.S & M & 45 & $\mathrm{nl}$ & $\downarrow$ & $\downarrow$ & 0 \\
\hline E.P.S & M & 46 & 0 & 0 & 0 & 0 \\
\hline S.B.S & $\mathbf{F}$ & 47 & $\downarrow$ & $\downarrow$ & $\mathrm{nl}$ & $\mathrm{nl}$ \\
\hline L.F.L & $\mathrm{F}$ & 49 & $\mathrm{nl}$ & $\mathrm{nl}$ & $\mathrm{nl}$ & $\mathrm{nl}$ \\
\hline S.N.P & $\mathrm{F}$ & 53 & 0 & 0 & 0 & 0 \\
\hline J.N & M & 59 & $\downarrow$ & $\mathrm{nl}$ & $\downarrow$ & $\mathrm{nl}$ \\
\hline G.M.M & M & 60 & $\downarrow$ & $\downarrow$ & $\downarrow$ & $\downarrow$ \\
\hline U.M.S & M & 61 & $\mathrm{nl}$ & $\mathrm{nl}$ & $\mathrm{nl}$ & $\mathrm{nl}$ \\
\hline E.S.C & M & 61 & $\downarrow$ & $\downarrow$ & nl & $\mathrm{nl}$ \\
\hline J.A.M & M & 62 & $\downarrow$ & $\downarrow$ & $\downarrow$ & $\downarrow$ \\
\hline M.A.O.B & $\mathrm{F}$ & 65 & $\downarrow$ & $\mathrm{nl}$ & $\downarrow$ & $\mathrm{nl}$ \\
\hline
\end{tabular}

M: masculino; F: feminino; nl: normorreflexia; 0: arreflexia; $\downarrow$ : hiporrefelexia; $\uparrow:$ hiperrelflexia 
ANEXO D

\section{VALORES DE $p$ NAS DIFERENTES CONDIÇÕES DE CONFLITO SENSORIAL, C1 a C6, DO ÍNDICE FINAL DE EQUILÍBRIO, CALCULADOS POR MEIO DO TESTE DE WILCOXON}

Tabela 1 - Valor de $p$ na Condição 1 da PDC nos Dias 60, 120, 180 e 365

\begin{tabular}{|c|c|c|c|c|c|c|c|c|c|}
\hline \multirow{3}{*}{$\begin{array}{l}\text { Condiçã } \\
\text { o de } \\
\text { conflito } \\
\text { sensorial }\end{array}$} & \multirow{3}{*}{$\begin{array}{c}\text { Estado } \\
\text { dos } \\
\text { pacientes }\end{array}$} & \multicolumn{8}{|c|}{ Resposta na PC nos dias } \\
\hline & & \multicolumn{2}{|c|}{60} & \multicolumn{2}{|c|}{120} & \multicolumn{2}{|c|}{180} & \multicolumn{2}{|c|}{365} \\
\hline & & $\mathbf{N}$ & $p$ & $\mathbf{N}$ & $p$ & $\mathbf{N}$ & $p$ & $\mathbf{N}$ & $p$ \\
\hline \multirow{3}{*}{ C1 } & pioraram & 10 & \multirow{3}{*}{0,837} & 14 & \multirow{3}{*}{0,648} & 11 & \multirow{3}{*}{0,824} & 15 & \multirow{3}{*}{0,898} \\
\hline & melhoraram & 10 & & 9 & & 8 & & 9 & \\
\hline & $\begin{array}{c}\text { sem } \\
\text { alterações }\end{array}$ & 4 & & 1 & & 5 & & 0 & \\
\hline
\end{tabular}

$\overline{\mathrm{PDC}}=$ Posturografia dinâmica computadorizada; $\mathrm{N}$ = quantidade de pacientes cuja C1 piorou, melhorou ou não se alterou; $p=p$-valor

Tabela 2 - Valor de $p$ na Condição 2 da PDC nos Dias 60, 120, 180 e 365

\begin{tabular}{|c|c|c|c|c|c|c|c|c|c|}
\hline \multirow{3}{*}{$\begin{array}{l}\text { Condição } \\
\text { de } \\
\text { conflito } \\
\text { sensorial }\end{array}$} & \multirow{3}{*}{$\begin{array}{c}\text { Estado } \\
\text { dos } \\
\text { pacientes }\end{array}$} & \multicolumn{8}{|c|}{ Resposta na PC nos dias } \\
\hline & & \multicolumn{2}{|c|}{60} & \multicolumn{2}{|c|}{120} & \multicolumn{2}{|c|}{180} & \multicolumn{2}{|c|}{365} \\
\hline & & $\mathbf{N}$ & $p$ & $\mathbf{N}$ & $p$ & $\mathbf{N}$ & $p$ & $\mathbf{N}$ & $p$ \\
\hline \multirow{3}{*}{ C2 } & pioraram & 11 & \multirow{3}{*}{0,794} & 8 & \multirow{3}{*}{0,345} & 14 & \multirow{3}{*}{0,531} & 10 & \multirow{3}{*}{0,681} \\
\hline & melhoraram & 10 & & 15 & & 7 & & 10 & \\
\hline & $\begin{array}{l}\text { sem } \\
\text { alterações }\end{array}$ & 3 & & 1 & & 3 & & 4 & \\
\hline
\end{tabular}

$\overline{\mathrm{PDC}}=$ Posturografia dinâmica computadorizada; $\mathrm{N}$ = quantidade de pacientes cuja C2 piorou, melhorou ou não se alterou; $p=\mathrm{p}$-valor 
Tabela 3 - Valor de p na Condição 3 da PDC nos Dias 60, 120, 180 e 365

\begin{tabular}{|c|c|c|c|c|c|c|c|c|c|}
\hline \multirow{3}{*}{$\begin{array}{l}\text { Condição } \\
\text { de } \\
\text { conflito } \\
\text { sensorial }\end{array}$} & \multirow{3}{*}{$\begin{array}{l}\text { Estado } \\
\text { dos } \\
\text { pacientes }\end{array}$} & \multicolumn{8}{|c|}{ Resposta na PC nos dias } \\
\hline & & \multicolumn{2}{|c|}{60} & \multicolumn{2}{|c|}{120} & \multicolumn{2}{|c|}{180} & \multicolumn{2}{|c|}{365} \\
\hline & & $\mathbf{N}$ & $p$ & $\mathbf{N}$ & $p$ & $\mathbf{N}$ & $p$ & $\mathbf{N}$ & $p$ \\
\hline \multirow{3}{*}{ C3 } & pioraram & 7 & \multirow{3}{*}{0,277} & 9 & \multirow{3}{*}{$0,048^{*}$} & 6 & \multirow{3}{*}{$0,020^{*}$} & 10 & \multirow{3}{*}{0,145} \\
\hline & melhoraram & 15 & & 14 & & 15 & & 14 & \\
\hline & $\begin{array}{c}\text { sem } \\
\text { alterações }\end{array}$ & 2 & & 1 & & 3 & & 0 & \\
\hline
\end{tabular}

$\overline{\mathrm{PC}}=$ Posturografia dinâmica computadorizada; $\mathrm{N}$ = quantidade de pacientes cuja C3 piorou, melhorou ou não se alterou; $p=\mathrm{p}$-valor; * valor apresenta significância estatística

Tabela 4 - Valor de $p$ na Condição 4 da PDC nos Dias 60, 120, 180 e 365

\begin{tabular}{|c|c|c|c|c|c|c|c|c|c|}
\hline \multirow{3}{*}{$\begin{array}{l}\text { Condição } \\
\text { de } \\
\text { conflito } \\
\text { sensorial }\end{array}$} & \multirow{3}{*}{$\begin{array}{l}\text { Estado } \\
\text { dos } \\
\text { pacientes }\end{array}$} & \multicolumn{8}{|c|}{ Resposta na PC nos dias } \\
\hline & & \multicolumn{2}{|c|}{60} & \multicolumn{2}{|c|}{120} & \multicolumn{2}{|c|}{180} & \multicolumn{2}{|c|}{365} \\
\hline & & $\mathbf{N}$ & $p$ & $\mathbf{N}$ & $p$ & $\mathbf{N}$ & $p$ & $\mathbf{N}$ & $p$ \\
\hline \multirow{3}{*}{ C4 } & pioraram & 2 & \multirow{3}{*}{$0,000^{*}$} & 4 & \multirow{3}{*}{$0,000^{*}$} & 3 & \multirow{3}{*}{$0,000^{*}$} & 1 & \multirow{3}{*}{$0,000^{*}$} \\
\hline & melhoraram & 21 & & 20 & & 19 & & 23 & \\
\hline & $\begin{array}{c}\text { sem } \\
\text { alterações }\end{array}$ & 1 & & 0 & & 2 & & 0 & \\
\hline
\end{tabular}

$\overline{\mathrm{PDC}}=$ Posturografia dinâmica computadorizada; $\mathrm{N}$ = quantidade de pacientes cuja C4 piorou, melhorou ou não se alterou; $p=\mathrm{p}$-valor; * valor apresenta significância estatística

Tabela 5 - Valor de $p$ na Condição 5 da PDC nos Dias 60, 120, 180 e 365

\begin{tabular}{|c|c|c|c|c|c|c|c|c|c|}
\hline \multirow{3}{*}{$\begin{array}{l}\text { Condição } \\
\text { de } \\
\text { conflito } \\
\text { sensorial }\end{array}$} & \multirow{3}{*}{$\begin{array}{l}\text { Estado } \\
\text { dos } \\
\text { pacientes }\end{array}$} & \multicolumn{8}{|c|}{ Resposta na PC nos dias } \\
\hline & & \multicolumn{2}{|c|}{60} & \multicolumn{2}{|c|}{120} & \multicolumn{2}{|c|}{180} & \multicolumn{2}{|c|}{365} \\
\hline & & $\mathbf{N}$ & $p$ & $\mathbf{N}$ & $p$ & $\mathbf{N}$ & $p$ & $\mathbf{N}$ & $p$ \\
\hline \multirow{3}{*}{ C5 } & pioraram & 6 & \multirow{3}{*}{0,433} & 3 & \multirow{3}{*}{$0,013^{*}$} & 1 & \multirow{3}{*}{$0,006^{\star}$} & 2 & \multirow{3}{*}{$0,004^{*}$} \\
\hline & melhoraram & 6 & & 11 & & 12 & & 12 & \\
\hline & $\begin{array}{c}\text { sem } \\
\text { alterações }\end{array}$ & 12 & & 10 & & 11 & & 10 & \\
\hline
\end{tabular}

$\overline{\mathrm{PDC}}$ = Posturografia dinâmica computadorizada; $\mathrm{N}$ = quantidade de pacientes cuja C5 piorou, melhorou ou não se alterou; $p=\mathrm{p}$-valor; * valor apresenta significância estatística 
Tabela 6 - Valor de $p$ na Condição 6 da PDC nos Dias 60, 120, 180 e 365

\begin{tabular}{|c|c|c|c|c|c|c|c|c|c|}
\hline \multirow{3}{*}{$\begin{array}{l}\text { Condição } \\
\text { de } \\
\text { conflito } \\
\text { sensorial }\end{array}$} & \multirow{3}{*}{$\begin{array}{l}\text { Estado } \\
\text { dos } \\
\text { pacientes }\end{array}$} & \multicolumn{8}{|c|}{ Resposta na PC nos dias } \\
\hline & & \multicolumn{2}{|c|}{60} & \multicolumn{2}{|c|}{120} & \multicolumn{2}{|c|}{180} & \multicolumn{2}{|c|}{365} \\
\hline & & $\mathbf{N}$ & $p$ & $\mathbf{N}$ & $p$ & $\mathbf{N}$ & $p$ & $\mathbf{N}$ & $p$ \\
\hline \multirow{3}{*}{ C6 } & pioraram & 5 & \multirow{3}{*}{0,328} & 4 & \multirow{3}{*}{0,124} & 2 & \multirow{3}{*}{$0,025^{\star}$} & 4 & \multirow{3}{*}{$0,015^{\star}$} \\
\hline & melhoraram & 6 & & 9 & & 10 & & 11 & \\
\hline & $\begin{array}{c}\text { sem } \\
\text { alterações }\end{array}$ & 13 & & 11 & & 12 & & 9 & \\
\hline
\end{tabular}

$\overline{\mathrm{PDC}}=$ Posturografia dinâmica computadorizada; $\mathrm{N}$ = quantidade de pacientes cuja C6 piorou, melhorou ou não se alterou; $p=\mathrm{p}$-valor; * valor apresenta significância estatística

Tabela 7 - Valor de p para o Índice Final de Equilíbrio da PDC nos Dias 60, 120,180 e 365

\begin{tabular}{|c|c|c|c|c|c|c|c|c|c|}
\hline \multirow{3}{*}{$\begin{array}{l}\text { Índice } \\
\text { final de } \\
\text { equilíbrio }\end{array}$} & \multirow{3}{*}{$\begin{array}{l}\text { Estado } \\
\text { dos } \\
\text { pacientes }\end{array}$} & \multicolumn{8}{|c|}{ Resposta na PC nos dias } \\
\hline & & \multicolumn{2}{|c|}{60} & \multicolumn{2}{|c|}{120} & \multicolumn{2}{|c|}{180} & \multicolumn{2}{|c|}{365} \\
\hline & & $\mathbf{N}$ & $p$ & $\mathbf{N}$ & $p$ & $\mathbf{N}$ & $p$ & $\mathbf{N}$ & $p$ \\
\hline \multirow{3}{*}{ IE } & pioraram & 8 & \multirow{3}{*}{$0,026^{*}$} & 4 & \multirow{3}{*}{$0,001^{*}$} & 4 & \multirow{3}{*}{$0,000^{*}$} & 2 & \multirow{3}{*}{$0,000^{*}$} \\
\hline & melhoraram & 12 & & 19 & & 19 & & 10 & \\
\hline & $\begin{array}{c}\text { sem } \\
\text { alterações }\end{array}$ & 4 & & 1 & & 1 & & 2 & \\
\hline
\end{tabular}

$\overline{\mathrm{PDC}}$ = Posturografia dinâmica computadorizada; $\mathrm{N}$ = quantidade de pacientes cujo IE piorou, melhorou ou não se alterou; $p=p$-valor; IE = Índice final de equilíbrio; * valor apresenta significância estatística 


\section{ANEXO E}

\section{VALORES DE $p$ NAS DIFERENTES CONDIÇÕES DE CONFLITO SENSORIAL, C1 A C6, E PARA O ÍNDICE FINAL DE EQUILÍBRIO, EM GRUPOS RESPOSTAS NEGATIVA E/OU POSITIVA PARA PDC NOS DIAS $0 \mathrm{E} / \mathrm{OU} 365$}

Tabela 1 - Valor de $p$ para as Condições 1 a 6 e IE ao comparar os grupos de pacientes com respostas negativa e positiva no Dia 0

\begin{tabular}{|c|c|c|c|}
\hline \multirow{2}{*}{ Variável } & \multicolumn{2}{|c|}{$\mathbf{N}$} & \multirow{2}{*}{$\boldsymbol{p}_{0}$ Wilcoxon } \\
\hline & Resposta negativa & Resposta positiva & \\
\hline C1 & 7 & 17 & $0,048^{*}$ \\
\hline $\mathrm{C2}$ & 7 & 17 & 0,172 \\
\hline C3 & 7 & 17 & 0,324 \\
\hline C4 & 7 & 17 & 0,061 \\
\hline C5 & 7 & 17 & $0,008^{*}$ \\
\hline C6 & 7 & 17 & $0,008^{*}$ \\
\hline IE & 7 & 17 & $0,004^{*}$ \\
\hline
\end{tabular}


Tabela 2 - Valor de $p$ para as Condições 1 a 6 e IE ao comparar os grupos com respostas negativa e positiva no Dia 365

\begin{tabular}{|c|c|c|c|}
\hline \multirow{2}{*}{ Variável } & \multicolumn{2}{|c|}{$\mathbf{N}$} & \multirow{2}{*}{$p_{365}{ }^{\text {Wilcoxor }}$} \\
\hline & Resposta negativa & Resposta positiva & \\
\hline C1 & 7 & 17 & 0,292 \\
\hline $\mathrm{C} 2$ & 7 & 17 & 0,324 \\
\hline C3 & 7 & 17 & 0,848 \\
\hline C4 & 7 & 17 & 0,204 \\
\hline C5 & 7 & 17 & $0,007^{*}$ \\
\hline C6 & 7 & 17 & $0,029^{*}$ \\
\hline IE & 7 & 17 & $0,024^{*}$ \\
\hline
\end{tabular}


Tabela 3 - Valor de $p$ para as Condições 1 a 6 e IE no grupo resposta negativa, comparando os Dias 0 e 365

\begin{tabular}{|c|c|c|c|}
\hline Variável & $\begin{array}{l}\text { Estado dos } \\
\text { pacientes }\end{array}$ & $\begin{array}{c}\mathbf{N} \\
\text { Resposta negativa }\end{array}$ & $p_{0,365}{ }^{\text {Wilcoxon }}$ \\
\hline & pioraram & 3 & \\
\hline \multirow[t]{3}{*}{ C1 } & melhoraram & 4 & 0,176 \\
\hline & sem alteração & 0 & \\
\hline & pioraram & 1 & \\
\hline \multirow[t]{3}{*}{ C2 } & melhoraram & 4 & 0,080 \\
\hline & sem alteração & 2 & \\
\hline & pioraram & 1 & \\
\hline \multirow[t]{3}{*}{ C3 } & melhoraram & 6 & $0,028^{*}$ \\
\hline & sem alteração & 0 & \\
\hline & pioraram & 0 & \\
\hline \multirow[t]{3}{*}{ C4 } & melhoraram & 7 & $0,018^{*}$ \\
\hline & sem alteração & 0 & \\
\hline & pioraram & 0 & \\
\hline \multirow[t]{3}{*}{ C5 } & melhoraram & 2 & 0,180 \\
\hline & sem alteração & 5 & \\
\hline & pioraram & 0 & \\
\hline \multirow[t]{3}{*}{ C6 } & melhoraram & 2 & 0,180 \\
\hline & sem alteração & 5 & \\
\hline & pioraram & 0 & \\
\hline \multirow[t]{2}{*}{ IE } & melhoraram & 7 & $0,018^{*}$ \\
\hline & sem alteração & 0 & \\
\hline
\end{tabular}

$\overline{\mathrm{N}}$ = quantidade de pacientes no grupo; $p_{0,365}{ }^{\text {wilcoxon }}=\mathrm{p}$-valor para os Dias 0 e 365 (teste de Wilcoxon); $\mathrm{C}=$ condição de conflito sensorial; IE = Índice final de equilíbrio; * = valor apresenta significância estatística 
Tabela 4 - Valor de $p$ para as Condições 1 a 6 e IE no grupo resposta positiva, comparando os Dias 0 e 365

\begin{tabular}{|c|c|c|c|}
\hline Variável & $\begin{array}{l}\text { Estado dos } \\
\text { pacientes }\end{array}$ & $\begin{array}{c}\text { N } \\
\text { Resposta positiva }\end{array}$ & $p_{0,365}{ }^{\text {Wilcoxon }}$ \\
\hline & pioraram & 12 & \\
\hline \multirow[t]{3}{*}{ C1 } & melhoraram & 5 & 0,356 \\
\hline & sem alteração & 0 & \\
\hline & pioraram & 9 & \\
\hline \multirow[t]{3}{*}{$\mathrm{C} 2$} & melhoraram & 6 & 0,477 \\
\hline & sem alteração & 2 & \\
\hline & pioraram & 9 & \\
\hline \multirow[t]{3}{*}{ C3 } & melhoraram & 8 & 0,794 \\
\hline & sem alteração & 0 & \\
\hline & pioraram & 2 & \\
\hline \multirow[t]{3}{*}{ C4 } & melhoraram & 15 & $0,005^{*}$ \\
\hline & sem alteração & 0 & \\
\hline & pioraram & 2 & \\
\hline \multirow[t]{3}{*}{ C5 } & melhoraram & 10 & $0,008^{*}$ \\
\hline & sem alteração & 5 & \\
\hline & pioraram & 4 & \\
\hline \multirow[t]{3}{*}{ C6 } & melhoraram & 9 & $0,033^{*}$ \\
\hline & sem alteração & 4 & \\
\hline & pioraram & 2 & \\
\hline \multirow[t]{2}{*}{ IE } & melhoraram & 13 & $0,005^{\star}$ \\
\hline & sem alteração & 2 & \\
\hline
\end{tabular}

$\overline{\mathrm{N}}=$ quantidade de pacientes no grupo; $p_{0,365}=\mathrm{p}$-valor para os Dias 0 e 365 (teste de Wilcoxon); $\mathrm{C}=$ condição de conflito sensorial; IE = Índice final de equilíbrio; * = valor apresenta significância estatística 
9 REFERÊNCIAS 


\section{REFERÊNCIAS ${ }^{1}$}

Abramides PA, Bento RF, Bitar RSM, Neto RVB, Tsuji RK. Como o implante coclear pode interferir na função vestibular? Arq Int Otorrinolaringol. 2009;13(2):195-200.

Arnesen AR. Fiber population of the vestibulococlear anastomosis in humans. Acta Otolaryngol. 1984;98:501-18.

Baloh, RW, Honrubia V. The central vestibular system, in Clinical neurophysiology of the vestibular system. $3^{\text {rd }}$ Editon. Oxford University Press: New York; 2001, p. 53-110.

Bance ML, O'Driscoll M, Giles E, Ramsden RT. Vestibular stimulation by multichannel cochlear implants. Laryngoscope. 1998;108(2):291-4.

Bittar RSM. Como o Posturografia Dinâmica Computadorizada pode nos Ajudar nos Casos de Tontura? Arq Int Otorrinolaringol. 2007a;11(3):330-3.

Bittar RSM, Simoceli L, Pedalini MEB, Bottino. Repercussão das medidas de correção das comorbidades no resultado da reabilitação vestibular. BJORL. 2007b;73(3):295-8.

Black FO. Effects of the auditory prosthesis on postural stability. Ann Otol Rhinol Laryngol. 1977a;86(Suppl 38):141-64.

Black FO. Present vestibular status of subjects implanted with auditory prothesis. Ann Otol Rhinol Laryngol 1977b;86(Suppl 38):49-56.

\footnotetext{
${ }^{1}$ Esta tese está de acordo com as seguintes normas, em vigor no momento desta publicação: Referências: adaptado de International Committee of Medical Journals Editors (Vancouver). Universidade de São Paulo. Faculdade de Medicina. Divisão de Biblioteca e Documentação. Guia de apresentação de dissertações, teses e monografias. Elaborado por Anneliese Carneiro da Cunha, Maria Julia de A. L. Freddi, Maria F. Crestana, Marinalva de Souza Aragão, Suely Campos Cardoso, Valéria Vilhena. 3a ed. São Paulo: Divisão de Biblioteca e Documentação; 2011. Abreviaturas dos títulos dos periódicos de acordo com List of Journals Indexed in Index Medicus.
} 
Black FO, Lilly DJ, Peterka RJ, Fowler LP, Simmons FB. Vestibulo-ocular and vestibulospinal function before and after cochlear implant surgery. Ann Otol Rhinol Laryngol Suppl. 1987;96(1 Pt 2 Suppl 128):106-8.

Black FO, Simmons FB, III Wall C. Human vestibulo-spinal responses to direct eletrical eighth nerve stimulation. Acta Otolaryngol. 1980;90:86-92.

Bonucci AS, Costa Filho OA, Mariotto LDF, Amantini RCB, Alvarenga KF. A função vestibular em indivíduos usuários de implante coclear. Rev Bras Otorrinolaringol. 2008;74(2):273-8.

Bouccara D, Estève Fraysse MJ, Loundon N, Fraysse B, Garabedian N, Sterkers O. Vestibular dysfunction after cochlear implantation: a national multicenter clinical study. Rev Laryngol Otol Rhinol. (Bord) 2005;126(4):275-8.

Brey RH, Facer GW, Trine MB, Lynn SG, Peterson AM, Suman VJ. Vestibular effects associated with implantation of multiple channel cochlear prosthesis. Am J Otol. 1995;16(4):424-30.

Buchman CA, Joy J, Hodges A, Telischi FF, Balkany TJ. Vestibular effects of cochlear implantation. Laryngoscope. 2004;114(10 Pt 2 Suppl 103):1-22.

Buckley KA, Tobey EA. Cross-modal plasticity and speech perception in preand postlingually deaf cochlear implant users. Ear Hear. 2011;32:2-15.

Burian M, Gstoettner W, Zundritsch R. Saccular afferent fibers to the cochlear nucleus in the guinea pig. Arch Otorhinolaryngol. 1989;246:23841.

Chiong CM, Nedzelski JM, Mcllmoyl LD, Shipp DB. Eletro-oculographic findings pre- and post-cochlear implantation. $J$ Otolaryngol. 1994;23(6):447-9.

Cohen NL, Hoffman RA. Complications of cochlear implant surgery in adults and children. Ann Otol Rhinol Laryngol. 1991;100:708-11. 
Curthoys, IS. Vestibular compensation and substitution. Curr Opin Neurol. 2000;13:27-30.

Doucet ME, Bergeron F, Lassonde M, Ferron P, Lepore F. Cross-modal reorganization and speech perception in cochlear implant users. Brain. 2006;129:3376-83.

Eisenberg LS, Nelson JR, House WF. Effects of the single-electrode cochlear implanto $\mathrm{n}$ the vestibular system of the profoundly deaf adult. Ann Otol Rhinol Laryngol Suppl. 1982;91(2 Pt 3):47-54.

Enticott JC, Tari S, Koh SM, Dowell RC, O'Leary SJ. Cochlear implant and vestibular function. Otol Neurotol. 2006;27(6):824-30.

Fayad JN, Linthicum FH. Multichannel Cochlear Implants: Relation of Histopathology to Performance. Laryngoscope. 2006;116:1310-20.

Filipo R, Patrizi M, La Gamma R, D’Elia C, La Rosa G, Barabara M. vestibular impairment and cochlear implantation. Acta Otolaryngol. 2006; 126(12):1266-74.

Fina M, Skinner M, Goebel JA, Piccirillo JF, Neely JG, Black O. vestibular dysfunction after cochlear implantation. Otol Neurotol. 2003;24(2):234-42.

Fukuda Y, Tangerina RP. Ação do implante coclear sobre o zumbido. RBMORL. 2000;7(1):3-7.

Furman JM, Baloh RW, Hain TC, Hirsch BE, Parker SW. Assessment: Electronystagmography. Report of the Therapeutics and Technology Assessment Subcommittee. Neurol. 1996;46(6):1763-6.

Goebel JA, Hanson JM, Langhofer LR, Fishel DG. Head-shake vestibuloocular reflex testing: comparison of results with rotational chair testing. Otolaryngol Head Neck Surg. 1995;112:203-9. 
Handzel O, Burgess BJ, Nadol JB. Histopathology of the peripheral vestibular System after cochlear implantation in the human. Otol Neurotol. 2005;27:57-64.

Herdman, SJ. Anatomy and Physiology of the normal vestibular system, in Vestibular rehabilitation, $3^{\text {rd }}$, Editor. 2007, Copyright: Philadelphia. p. 2-18.

Hansen S, Anthonsen K, Stangerup SE, Jensen JH, Thomsen J, CayéThomasen P. Unexpected findings and surgical complications in 505 consecutive cochlear implantations: a proposal for reporting consensus. Acta Oto-Laryngologica. 2010;130:540-9.

Himi T, Shintani T, Yamaguchi T, Harabuchi Y, Kataura A. Vestibular function in cochlear implants-prognostic factors and postoperative damage. Nippon Jibiinkoka Gakkai Kaiho. 1995;98(7):1111-8.

Huygen PL, Hinderink JB, Van Den Broek P, Van Den Borne S, Brokx JP, Mens LH, Admiraal RJ. The risk of vestibular function loss after intracochlear implantation. Acta Otolaryngol Suppl. 1995;Pt2:270-2.

Huygen PL, Van Den Broek P, Spies TH, Mens LH, Admiraal RJ. Does intrcochlear implantation jeopardize vestibular function? Ann Otol Rhinol Laryngol. 1994;103(8 Pt 1):609-14.

Ito $\mathrm{J}$. Influence of the multichannel cochlear implant on vestibular function. Otolaryngol Head Neck Surg. 1998;118(6):900-2.

Ito J. Tinnitus suppression in cochlear implant patients. Otolaryngol Head Neck Surg. 1997;117:701-3.

Ito J, Sakakihara J. Suppression of tinnitus by cochlear implantation. Am J Otolaryngol. 1994;15(2):145-8.

Jacot E, Van Den Abbeele T, Debre HR, Wiener-Vacher SR. Vestibular impairments pre-and post-cochlear implant in children. Int $\mathrm{J}$ Pediatr Otorhinolaryngol. 2009;73(2):209-17. 
Kandel ER, Schwartz JH, Jessel TM. O Sistema vestibular. In: Goldberg ME, Hudspeth AJ. Princípios da Neurociência. São Paulo: Manole; 2003 p. 801-15.

Kim HS, Kim DI, Chung IH, Lee WS, Kim KY. Topographical relationship of the facial and vestibulocochlear nerves in the subarachnoid space and internal auditory canal. AJNR Am. J. Neuroradiol. 1998;19:1155-61.

Kim DK, Bae SC, Park KH, Jun BC, Lee DH, Yeo SW, Park SN. Tinnitus in patients with profound hearing loss and the effect of cochlear implantation. Eur Arch Otorhinolaryngol. 2013;270(6):1803-8.

Krause E, Wechtenbruch J, Rader T, Berghaus A, Gürkov R. Impaired fixation suppression is a risk factor for vertigo after cochlear implantation. J Laryngol Otol. 2009a;123:845-50.

Krause E, Louza JPR, Hempel JM, Wechtenbruch J, Gürkov R. Influence of cochlear implantation on peripheral vestibular receptor function. Otolaryngol Head Neck Surg. 2010;142(6):809-13.

Krause E, Louza JPR, Hempel JM, Wechtenbruch J, Rader T, Gürkov R. Prevalence and Characteristics of Preoperative Balance Disorders in Cochlear Implant Candidates. Ann Otol Rhinol Laringol. 2008;117(10):764-8.

Krause E, Louza JPR, Hempel JM, Wechtenbruch J, Rader T, Gürkov R. Effect of cochlear implantation on horizontal semicircular canal function. Eur Arch Otorhinolaryngol 2009b; 266: 811-817.

Krause E, Louza JPR, Hempel JM, Wechtenbruch J, Rader T, Gürkov R. Incidence and quality of vertigo symptoms after cochlear implantation. $J$ Laryngol Otol 2009c; 123: 278-282.

Krause E, Wechtenbruch J, Rader T, Gürkov R. Influence of cochlear implantation on sacculus function. Otolaryngol Head Neck Surg. 2009d;140(1):108-13. 
Kubo T, Yamamoto K, Iwaki T, Doi K, Tamura M. Different forms of dizziness occurring after cochlear implant. Eur Arch Otorhinolaryngol. 2001;258(1): $9-12$.

Kubo T,Yamamoto K, Mamoto Y, Iwaki T. Significance of vestibular function in cochlear implant patients. Adv Otorhinolaryngol. 2000;57:176-9.

Lacour M, Dutheil S, Brahim T, Lopez C, Borel L. Tell me your Vestibular Deficit, and I'll tell you how you'll compensate. Ann NY Acad Sci. 2009;1164:268-78.

Lacour M. Restoration of vestibular function: basic aspects and practical advances for rehabilitation. Curr Med Res. 2006;22(9):1651-9.

Lee DS, Lee JS, Oh SH, Kim SK, Kim JW, Chung JK, et al. cross-modal plasticity and cochlear implants. Nature. 2001;409:149-50.

Limb CJ, Francis HF, Lustig LR, Niparko JK, Jammal H. Benign positional vertigo after cochlear implantation. Otolaryngol Head Neck Surg. 2005; 132(5):741-5.

Lohle E, Frischmuth S, Holm M, Becker L, Flamm K, Laszig R, Beck CHL, Lehnhardt T. Speech recognition, speech production and speech intelligibility in children with hearing aides versus implanted children. Int $\mathrm{J}$ Ped Otorhinolaringol. 1999;47:165-9.

Lomber SG, Meredith MA, Kral A. Cross-modal plasticity in specific auditory cortices underlies visual compensations in the deaf. Nat Neurosci. 2010;13:1421-7.

Magnusson M, Petersen H, Harris S, Johansson R. Postural Control and Vestibular Function in Patients Selected for Cochlear Implantation. Acta Otolaryngol (Stockh). 1995;Suppl 520:277-8.

Mangham CA. Effects of cochlear prostheses on vestibule-ocular reflexes to rotation. Ann Otol Rhinol laryngol Suppl. 1987;128:101-4. 
Melvin TN, Della Santina CC, Carey JP, Migliaccio AA. The effects of cochlear implantation on vestibular function. Otol Neurotol. 2008;30:87-94.

McCabe BF, Ryu JH, Sekitani T. Further experiments on vestibular compensation. Laryngoscope. 1972;82(3):381-96.

Migliaccio AA, Della Santina CC, Carey JP, Niparko JK, Minor LB. The vestíbulo-ocular reflex response to head impulses rarely decreases after cochlear implantation. Otol Neurotol. 2005;26(4):655-60.

Nageris B, Braverman I, Kalmanowitz M, Segal K, Frenkiel S. Connections of the facial and vestibular nerves: an anatomic study. J Otolaryngol. 2000;29:159-61.

Newlands SD, Dara S, Kaufman GD. Relationship of static and dynamic mechanisms in vestibule-ocular reflex. Laryngoscope. 2005;115:191-204.

Nita LM, Lorenzetti FTM, Sanchez TG, Brito RV, Bento RF. Zumbido em Pacientes Submetidos a Implante Coclear: Frequência de Ocorrência e Evolução. Int Arch Otorhinolaryngol. 2002:6(3):195-200.

Oliveira JAA, Bernal TMO. Ototoxicidade de aminoglicosídeos e otoproteção. In: Campos CAH, Costa HOO. Tratado de Otorrinolaringologia. São Paulo: Roca; 2002. p. 148-167. v1.

Osberger MJ, Zimmerman-Phillips SZ, Barker M, Geier L. Speech recognition performance o folder children with cochlear implant. Am J Otol. 1998;19:152-7.

Özdogmus O, Sezen O, Kubilay U, Saka E, Duman U, San T, Çavdar S. Connections between the facial, vestibular and cochlear nerve bundles within the internal auditory canal. J Anat. 2004;205:65-75.

Palau EM, Gil JLM, Vidal CM, González JCF, Cabrera OA, Macías AR. Acúfeno e implante coclear. Experiencia preliminar. Acta Otorrinolaringol Esp. 2010;61(6):405-11. 
Pierchala K. The study of the vestibular function in cochlear implant patients. Otolaryngol Pol. 1996;50(3):281-5.

Rasmussen AT. Studies of the VIII'th cranial nerve of man. Laryngoscope. 1940;50:67-83.

Ribari O, Kustel M, Szirmai A, Repassy G. Cochlear implantation influences contralateral hearing and vestibular responsiveness. Acta Otolaryngol. 1999;119(2):225-8.

Ribari O, Szirmai A, Kustel M, Repassy G. How does cochlear implantation affect the contralateral vestibular system? Int Tinnitus J. 2002;8(2):108-10.

Rossi G, Solero P, Rolando M, Spadola Bisetti M. Vestibular function and cochlear implant. ORL J Otorhinolaryngol Relat Spec. 1998;60(2):85-7.

Schwab B, Durisin M, Kontorini G. Investigation of balance function using dynamic posturography under electrical-acoustic stimulation in cochlear implant recipients. Int J Otolaryngol. 2010;2010:1-7.

Silverstein H. Cochlear and vestibular Gross and histologic anatomy (as seen from post auricular approach). Otolaryngol Head Neck Surg. 1984;92:20711.

Steenerson RL, Cronin GW, Gary LB. Vertigo after cochlear implantation. Otol Neurotol. $2001 ; 22(6): 842-3$.

Suarez H, Angeli S, Suarez A, Rosales B, Carrera X, Alonso R. Balance sensory organization in children with profound hearing loss and cochlear implants. Int J Pediatr Otorhinolaryngol. 2007;71(4):629-37.

Szirmai A, Ribari O, Repassy G. Air caloric computer system application in monitoring vestibular function changes after cochlear implantation. Otolaryngol Head Neck Surg. 2001;125(6):631-4.

Tien HC, Linthicum FH. Histopathologic changes in the vestibule after cochlear implantation. Otolaryngol Head Neck Surg. 2002;127(4):260-4. 
Todt I, Basta D, Ernst A. Does the surgical approach in cochlear implantation influence the occurence of postoperative vertigo? Otolaryngol Head Neck Surg. 2008;138(1):8-12.

Vibert D, Häusler R, Kompis M, Vischer M. Vestibular function in patients with cochlear implantation. Acta otolaryngol Suppl. 2001;545:29-34.

Viccaro M, Mancini P, La Gamma R, De Seta E, Covelli E, Filipo R. Positional vertigo and cochlear implantation. Otol Neurotol. 2007;28960:764-7.

Zanetti D, Campovecchi CB, Balzanelli C, Pasini S. Paroxysmal positional vertigo after cochlear implantation. Acta Otolaryngol. 2007;127(5):452-8. 
APÊNDICES 


\section{APÊNDICE 1 \\ CARTA DA COMISSÃO DE ÉTICA}

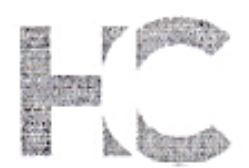

\section{APROVAÇÃO}

A Comissão de Ética para Análise de Projetos de Pesquisa CAPPesq da Diretoria Clínica do Hospital das Clínicas e da Faculdade de Medicina da Universidade de São Paulo, em sessão de 20/02/2008, APROVOU o Protocolo de Pesquisa $n^{\circ}$ 0983/07, intitulado: "AVALIAÇÃo SEQUENCIAL DO EQUILÍBRIO PÓS-IMPLANTE COCLEAR EM PACIENTES PÓS-LINGUAIS " apresentado pelo Departamento de OFTALMOLOGIA E OTORRINOLARINGOLOGIA, inclusive o Termo de Consentimento Livre e Esclarecido.

Cabe do pesquisador elaborar e apresentar à CAPPesq. os relatórios parciais e final sobre a pesquisa (Resolução do Conselho Nacional de Saúde n० 196, de 10/10/1996, inciso IX.2, letra "c").

Pesquisador (a) Responsável: Dra Roseli Saraiva Moreira Bittar

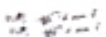

Pesquisador (a) Executante: Patricia Nunes Arena

CAPPesq, 22 de Fevereiro de 2008

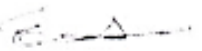

Prof. Dr. Eduardo Massad Presidente da Comissão de Ética para Análise de Projetos de Pesquisa 


\section{APÊNDICE 2}

HOSPITAL DAS CLÍNICAS

DA

FACULDADE DE MEDICINA DA UNIVERSIDADE DE SÃO PAULO

\section{TERMO DE CONSENTIMENTO LIVRE E ESCLARECIDO}

(Instruções para preenchimento no verso)

\section{I - DADOS DE IDENTIFICAÇÃO DO SUJEITO DA PESQUISA OU RESPONSÁVEL LEGAL}

1. NOME DO PACIENTE

DOCUMENTO DE IDENTIDADE № : GÊNERO: M Ž F Ž

DATA NASCIMENTO:

ENDEREÇO № APTO:

BAIRRO:

CIDADE

CEP: TELEFONE: DDD (........)

2.RESPONSÁVEL LEGAL:

NATUREZA (grau de parentesco, tutor, curador, etc.):

DOCUMENTO DE IDENTIDADE: GÊNERO: MŽ $F \check{Z}$ DATA NASCIMENTO:

ENDEREÇO: № APTO:

BAIRRO: CIDADE:

CEP: TELEFONE: DDD (......).

\section{II - DADOS SOBRE A PESQUISA CIENTÍFICA}

1. TíTULO DO PROTOCOLO DE PESQUISA: Avaliação sequencial do equilíbrio pósimplante coclear em pacientes pós-linguais PESQUISADOR RESPONSÁVEL: Dra. Roseli Saraiva Moreira Bittar CARGO/FUNÇÃO: Médico Assistente INSCRIÇÃO CONSELHO REGIONAL: № 39729 UNIDADE DO HCFMUSP: Disciplina de Otorrinolaringologia 
2. AVALIAÇÃO DO RISCO DA PESQUISA:

\begin{tabular}{|c|c|c|}
\hline SEM RISCO $\square$ & RISCO MÍNIMO 区 & RISCO MÉDIO $\square$ \\
\hline RISCO BAIXO & RISCO MAIOR $\square$ & \\
\hline
\end{tabular}

3. DURAÇÃO DA PESQUISA: 2 anos

\section{III - REGISTRO DAS EXPLICAÇÕES DO PESQUISADOR AO PACIENTE OU SEU REPRESENTANTE LEGAL SOBRE A PESQUISA CONSIGNANDO:}

1. Justificativa e os objetivos da pesquisa:

Nossa pesquisa pretende verificar se o uso do implante coclear produz efeitos benéficos no seu organismo em relação ao equilíbrio.

2. Procedimentos que serão utilizados e propósitos, incluindo a identificação dos procedimentos que são experimentais:

O senhor(a) vai realizar 3 exames de equilíbrio (Eletronistagmografia, Posturografia e Cadeira Rotatória) para saber como está o seu labirinto. A Eletronistagmografia visa avaliar os movimentos oculares (utilizando uma barra fixa com luzes) e o labirinto através da irrigação das orelhas com água quente $\left(44^{\circ} \mathrm{C}\right)$ e depois fria $\left(30^{\circ} \mathrm{C}\right)$ por 40 segundos. A Posturografia é um exame onde o(a) senhor(a) ficará em cima de uma plataforma que oscila e registra o seu equilíbrio. O exame da Cadeira Rotatória é feito com o(a) senhor(a) sentado (a) em uma cadeira que oscila e registra também o equilíbrio. Todos os dados dos exames vão para um computador e são analisados. Os exames são realizados 1 dia antes da cirurgia de implante coclear. Após a ativação do implante coclear os exames são realizados novamente com 30, 120, 180 e 365 dias. O(a) senhor(a) será acompanhado o tempo todo pelo examinador.

3. Desconfortos e riscos esperados:

Depois dos exames e dos exercícios o(a) senhor(a) pode experimentar um pouco de tontura que é normal e passa rapidamente.

4. Benefícios que poderão ser obtidos:

A melhora importante de sua tontura.

IV - ESCLARECIMENTOS DADOS PELO PESQUISADOR SOBRE GARANTIAS DO SUJEITO DA PESQUISA CONSIGNANDO:

1. Acesso, a qualquer tempo, às informações sobre procedimentos, riscos e benefícios relacionados à pesquisa, inclusive para eliminar eventuais dúvidas: o(a) senhor(a) poderá ver seu prontuário e fazer perguntas sobre a pesquisa durante todo o processo de tratamento.

2. Liberdade de retirar seu consentimento a qualquer momento e de deixar de participar do estudo, sem que isto traga prejuízo à continuidade da assistência: em qualquer momento que o(a) senhor(a) queira, poderá desistir do projeto sem que isso lhe traga qualquer malefício. 
3. Salvaguarda da confidencialidade, sigilo e privacidade: o seu nome será mantido em segredo durante todo o tratamento e não serão divulgadas informações a respeito do seu caso.

4. Disponibilidade de assistência no HCFMUSP, por eventuais danos à saúde, decorrentes da pesquisa: se, porventura, o(a) senhor(a) achar que a pesquisa o prejudicou de alguma maneira, além de poder desistir o senhor terá todo o auxílio necessário dentro do Hospital das Clínicas.

5. Viabilidade de indenização por eventuais danos à saúde decorrentes da pesquisa: no caso de ocorrer qualquer dano à sua saúde, está prevista em lei a indenização e acompanhamento médico vitalício.

\section{INFORMAÇÕES DE NOMES, ENDEREÇOS E TELEFONES DOS RESPONSÁVEIS PELO ACOMPANHAMENTO DA PESQUISA, PARA CONTATO EM CASO DE INTERCORRÊNCIAS CLÍNICAS E REAÇÕES ADVERSAS}

Dra. Patrícia Nunes Arena

Av. Dr. Enéas de Carvalho Aguiar, 255

Instituto Central $-6^{\circ}$ andar - Departamento de Otorrinolaringologia

São Paulo - 05403-010

Fone: $3069-6538$

\section{OBSERVAÇÕES COMPLEMENTARES}

\section{CONSENTIMENTO PÓS-ESCLARECIDO}

Declaro que, após convenientemente esclarecido pelo pesquisador e ter entendido

o que me foi explicado, consinto em participar do presente Protocolo de Pesquisa

São Paulo, de de $20 \ldots . .$. 